\title{
WILEY-VCH
}

\section{Tracking Structural Phase Transitions in Lead Halide Perovskites by means of Thermal Expansion}

Masoumeh Keshavarz*, Martin Ottesen, Steffen Wiedmann, Michael Wharmby, Robert Küchler, Haifeng Yuan, Elke Debroye, Julian A. Steele, Johan Martens, Nigel Hussey, Martin Bremholm, Maarten B. J. Roeffaers and Johan Hofkens*

Dr. Masoumeh Keshavarz, Dr. Haifeng Yuan, Dr. Elke Debroye and Prof. Johan Hofkens, Molecular Imaging and Photonics, Department of Chemistry, Katholieke Universiteit Leuven, Celestijnenlaan 200F, 3001 Leuven, Belgium.

Martin Ottesen and Dr. Martin Bremholm, Department of Chemistry and iNANO, Aarhus University, Langelandsgade 140, 8000 Aarhus C, Denmark.

Dr. Steffen Wiedmann and Prof. Nigel Hussey, High Field Magnet Laboratory and Institute for Molecules and Materials, Radboud University, Toernooiveld 7, 6525 ED Nijmegen, Netherlands.

Dr. Michael Wharmby, Deutsches Elektronen-Synchrotron (DESY), Notkestr. 85, 22607 Hamburg, Germany.

Dr. Robert Küchler, Max Planck Institute for Chemical Physics of Solids, Nöthnitzer Str. 40, 01187 Dresden, Germany.

Dr. Julian A. Steele, Prof. Johan Martens and Prof. Maarten B. J. Roeffaers, Department of Microbial and Molecular Systems, Centre for Surface Chemistry and Catalysis (COK), KU Leuven, Celestijnenlaan 200F, 3001 Leuven, Belgium.

E-mail: masoumeh.keshavarz@kuleuven.be and johan.hofkens@kuleuven.be

Keywords: perovskite, structural phase transition, thermal expansion 


\section{WILEY-VCH}

The extraordinary properties of lead-halide perovskite materials have spurred intense research since they have a realistic perspective to play an important role in future photovoltaic devices. It is known that they undergo structural phase transitions as a function of temperature that firmly alter their optical and electronic properties. Yet, their precise phase transition temperature and exact crystal structure in each phase are controversially discussed in the literature. We have measured the linear thermal expansion $\Delta L(T) / L$ of single crystals of $\mathrm{APbX}_{3}(\mathrm{~A}=\mathrm{MA}, \mathrm{FA} ; \mathrm{X}=$ $\mathrm{I}, \mathrm{Br}$ ) below room temperature using a high-resolution capacitive dilatometer to accurately determine the phase transition temperatures. For $\delta-\mathrm{FAPbI}_{3}$, we discover two wide regions of negative thermal expansion below 173 and $54 \mathrm{~K}$, and a cascade of sharp transitions for $\mathrm{FAPbBr}_{3}$ that have neither been reported nor identified until now. We identified their crystal phases via powder X-ray diffraction. Moreover, we exemplify that transport under steady-state illumination is considerably altered at the structural phase transition in the MA compounds. Our results provide advanced insights into the evolution of the crystal structure with decreasing temperature that are essential to interpret the growing interest in investigating the electronic, optical and photonic properties of lead-halide perovskite materials. 


\section{WILEY-VCH}

The success of hybrid organic-inorganic perovskites (HOIP) in photovoltaic applications is based on their high power conversion efficiencies, up to $23.3 \%$, in combination with seemingly low production costs. ${ }^{[1-4]}$ Owing to their remarkable photo-physical properties such as low exciton binding energies, ${ }^{[5,6]}$ high absorption coefficients, ${ }^{[7]}$ extended carrier lifetimes and diffusion lengths, ${ }^{[8,9]}$ HOIPs are considered as candidate materials for the next generation of photovoltaic devices.

The basic formula unit of the HOIPs is $\mathrm{ABX}_{3}$, where $A$ is the organic ammonium cation (MA: $\mathrm{CH}_{3} \mathrm{NH}_{3}{ }^{+}$or $\mathrm{FA}: \mathrm{NH}_{2} \mathrm{CH}=\mathrm{NH}_{2}{ }^{+}$), $B$ is the inorganic cation (here $\mathrm{Pb}$ ) and $X$ is the halide (here $\mathrm{Br}$ or I). In view of the intense ongoing investigation of the electronic and optical properties, such as temperature-dependent charge carrier dynamics and intrinsic scattering processes, it is of fundamental interest to precisely explore the evolution of the perovskite crystal structure with temperature. In order to elucidate the miscellany of the so far reported crystal phases and transition temperatures, in particular in the FA-based HOIPs, we use thermal expansion to accurately determine the temperature evolution of the crystal structure. A change in the crystal structure has severe consequences for the electronic properties of each material including changes in the band structure and thus in the band gap, which consequently alters their optical properties. ${ }^{[10,11]}$ Furthermore, a structural phase transition may also lead to changes in the phonon structure that in turn influences the exciton binding energy due to a change in the dielectric constant. ${ }^{[12-14]}$ Another important question that arises is whether and how the charge transport properties under steady-state illumination are altered, and if the temperaturedependent charge carrier mobility is still a good means of identifying the underlying scattering mechanisms of the charge carriers by phonons. ${ }^{[15,16]}$

Long before lead halide perovskites were identified as candidate materials for photovoltaic applications, their structural phase transitions and corresponding entropies were investigated using temperature-dependent X-ray diffraction (XRD), ${ }^{[17]}$ measurements of the temperaturedependent complex permittivity ${ }^{[17]}$ as well as calorimetric and spectroscopic studies, ${ }^{[18]}$ with a 


\section{WILEY-VCH}

focus on the dynamic disorder of the methylammonium group within the high-temperature phase(s). The structural phase transitions found in Refs. $[17,18]$ in $\mathrm{MAPbI}_{3}$ and $\mathrm{MAPbBr}_{3}$ are summarized in Table SI in the Supporting Information.

In this work, we measure the linear thermal expansion $\Delta L / L$ of the tri-halide perovskites $\mathrm{MAPbI}_{3}, \delta-\mathrm{FAPbI}_{3}, \mathrm{MAPbBr}_{3}$ and $\mathrm{FAPbBr}_{3}$ using capacitive dilatometry between 4.2 and 280 K. In $\mathrm{MAPbI}_{3}$ and $\mathrm{MAPbBr}_{3}$, we determine all phase transition temperatures until we reach the orthorhombic phase at low temperatures. The linear thermal expansion in $\delta-\mathrm{FAPbI}_{3}$ and $\mathrm{FAPbBr}_{3}$ is found to be extremely complex compared to their MA counterparts. $\mathrm{FAPbBr}_{3}$ exhibits several sharp transitions between 120 and $160 \mathrm{~K}$ while $\delta$-FAPbI 3 is distinct and shows negative linear thermal expansion across temperature intervals below 173 and 54 K. Singlecrystal and powder X-ray diffraction (SCXRD, and PXRD) were used to follow the evolution of the lattice parameters. SCXRD verified both the overall trend and the large thermal expansion observed in both compounds. Via PXRD, we determined the change of the crystal phase in $\delta-\mathrm{FAPbI}_{3}$ at $173 \mathrm{~K}$ from the space group $P 6_{3} / m m c$ to $P 6_{3} / m$. For $\mathrm{FAPbBr}_{3}$, a decrease in symmetry has been found in both PXRD and SCXRD upon cooling from $300 \mathrm{~K}$ to $100 \mathrm{~K}$. Two phase transitions have been observed for $\mathrm{FAPbBr}_{3}$ at $250 \mathrm{~K}$ and $165 \mathrm{~K}$ from cubic to tetragonal and tetragonal to orthorhombic, respectively. Structural phase transitions in general affect not only the optical and photonic response, ${ }^{[10-14]}$ but also charge transport under steadystate illumination as we demonstrate in subsequent transport measurements on $\mathrm{MAPbI}_{3}$ and $\mathrm{MAPbBr}_{3}$.

The volume thermal expansion coefficient $\beta=-1 / V(\partial S / \partial T)_{P}$, where $S$ denotes the entropy at constant pressure $P$, is a measure of the relative change in a sample's volume, $V$, and is a powerful tool to probe various kinds of phase transitions. Compared to other thermodynamic probes, such as heat capacity, thermal expansion quantifies the directional dependence of the crystal structure by measuring the linear thermal expansion $\Delta L(T) / L$ or the linear coefficient $\alpha=-1 / L(d L / d T)_{P}$ along different crystallographic axes. For all halide perovskite materials 


\section{WILEY-VCH}

under investigation, we have performed temperature sweeps on different bulk single crystals, determined $\Delta L(T) / L,{ }^{[19,20]}$ and normalized it to the reference temperature e.g. at $280 \mathrm{~K}$, i.e. $\Delta L / L$ $(280 \mathrm{~K})=0$.

Let us first focus on the MA-based lead halide perovskite materials, to date the most studied compounds in the tri-halide perovskite family. The results of the thermal expansion using capacitive dilatometry are shown in Figure 1. We first investigate $\mathrm{MAPbI}_{3}$. Figure 1(a) shows the linear thermal expansion, $\Delta L / L$, and the linear thermal expansion coefficient, $\alpha$, as a function of temperature $T . \Delta L / L$ increases monotonically in the entire temperature range but exhibits a sharp step at $T^{P}=160.9 \mathrm{~K}$ that we identify as the orthorhombic to tetragonal phase transition, see inset of Figure 1(a). ${ }^{[21-23]}$ In Figure 1(b), we show the longitudinal resistance $R_{x x}$, measured in a Hall-bar geometry, of a $\mathrm{MAPbI}_{3}$ single crystal under steady-state illumination as a function of temperature (details see experimental section). The resistance decreases by a factor of two from room temperature down to $161 \mathrm{~K}$. Precisely at $T^{P}, R_{x x}$ abruptly rises by one order of magnitude before decreasing again markedly with decreasing temperature in the orthorhombic regime. The sharpness of the transition observed in transport is reminiscent of the one observed in linear thermal expansion. However, the behavior of $R_{x x}$ with decreasing temperature deserves particular attention. Firstly, below $T^{P}, R_{x x}$ decreases gradually with temperature down to $80 \mathrm{~K}$ and then appears to saturate. Secondly, as indicated by the dashed line in Figure 1(b), we find that the overall decrease of $R_{x x}$ in temperature is metallic $\left(\mathrm{d} R_{x x} / \mathrm{d} T>0\right)$ down to $25 \mathrm{~K}$ if one neglects the feature associated with the structural phase transition. Below $25 \mathrm{~K}, R_{x x}$ exhibits an upturn, which is attributed to thermally activated hopping of charge carriers. ${ }^{[16]}$ The transition from the tetragonal to the cubic phase occurs above $320 \mathrm{~K},{ }^{[18]}$ also recently investigated by a thermomechanical analysis. ${ }^{[24]}$

We now turn to the experimental results obtained for $\mathrm{MAPbBr}_{3}$ that are presented in Figure 1(c). Compared to $\mathrm{MAPbI}_{3}$, the change of the halide from I to $\mathrm{Br}$ has consequences on the 


\section{WILEY-VCH}

observed structural phase transitions that, as discussed in the Supporting Information, also depend on the quality of the crystals exemplified with two batches we refer to as batch A and B (with a presumably higher defect concentration, see Supporting Information). $\mathrm{MAPbBr}_{3}$ is known to exhibit three structural phase transitions in the temperature range between 4.2 and $280 \mathrm{~K}$ as summarized in Table SI and Refs. [17,18]. The $T$-dependence of the linear thermal expansion and the thermal expansion coefficient $\alpha$ for batch A are illustrated in Figure 1(c). $\Delta L / L$ first slowly increases up to around $147 \mathrm{~K}$ with increasing $T$. Then, two steps in $\Delta L / L$ occur at 149.3 and $154.9 \mathrm{~K}$. These steps appear as maxima in $\alpha(T)$, shown in Figure $1(\mathrm{~d}-\mathrm{f})$, and are identified as transitions from the orthorhombic (OR) to the tetragonal-II (TG (II)) phase at $T^{P}$ $=149.3 \mathrm{~K}$, and from the tetragonal-II to the tetragonal-I (TG (I)) phase at $T^{P}=154.9 \mathrm{~K}$, respectively. ${ }^{[17,18]}$ In the tetragonal-I phase, $\Delta L / L$ monotonically increases with temperature followed by a strong pronounced increase at $235 \mathrm{~K}$, which we identify as the transition from the tetragonal-I to the high-temperature cubic phase. For $T>235 \mathrm{~K}, \Delta L / L$ continues to increase with increasing temperature. Samples from batch B exhibit a different behavior as shown in the Supporting Information (Figures S1 and S2). Based on this observation, it would appear that disorder plays an important role in the underlying crystal structure that also alters the electronic (see Figure S2) and optical properties of $\mathrm{MAPbBr}_{3}$.

Having demonstrated that linear thermal expansion is a powerful technique to probe the evolution of the crystal structure, and to precisely determine phase transition temperatures, we now turn our attention to the FA-based lead halide perovskite materials. In contrast to the MAbased HOIPs, there are only a few experimental studies that address the low-temperature structural phase transitions in FA compounds. We show that the change from MA to the smaller organic ammonium cation, FA, results in profound changes in the linear thermal expansion. The results obtained for $\delta-\mathrm{FAPbI}_{3}$ and $\mathrm{FAPbBr}$ are presented in figures 2 and 3 with the phase transition temperatures and phases specified in Tables I and II, respectively. It should be noted that previous reports on the crystal structure derived from variable temperature single 


\section{WILEY-VCH}

crystal/powder XRD studies of $\mathrm{FAPbBr}_{3}$ are contradictory. ${ }^{[27,28]}$ We first focus on $\delta-\mathrm{FAPbI}_{3}$ in Figure 2(a). In contrast to all other materials investigated here, $\delta-\mathrm{FAPbI}_{3}$ does not exhibit abrupt transitions in $\Delta L(T) / L$. Instead, two regions of negative thermal expansion (NTE), highlighted by the boxes in Figure 2(a), are found that are superimposed on a positive thermal expansion background with decreasing temperature. The first region of NTE is found below $T^{P}=173 \mathrm{~K}$, the second is observed below $54.5 \mathrm{~K}$ after which $\Delta L / L$ considerably increases with decreasing temperature. Both transitions are also clearly identified in $\alpha(T)$.

Negative thermal expansion is generally associated with materials that exhibit ferroelectric, magnetic or charge-transfer phase transitions that cause the material to contract upon increasing temperature due to either redistribution or ordering of ferro-electric/-magnetic domains. ${ }^{[29]}$ The first region of NTE in $\delta-\mathrm{FAPbI}_{3}$ is found between 173 and $150 \mathrm{~K}$ with a thermal expansion coefficient $\alpha \approx-1.3 \cdot 10^{-4} \mathrm{~K}^{-1}$. The low-temperature NTE exhibits a remarkably large value of $\alpha$ $\approx-2.5 \cdot 10^{-4} \mathrm{~K}^{-1}$ that compares to values in the order of $-2.5 \cdot 10^{-5} \mathrm{~K}^{-1}$ in Ge-doped anti-perovskite manganese nitrates ${ }^{[30]}$ and $-1.15 \cdot 10^{-4} \mathrm{~K}^{-1}$ in reduced layered ruthenates. ${ }^{[31]}$ Recent reports on the low temperature phase behaviour of $\delta-\mathrm{FAPbI}_{3}$ are summarized in Table $\mathrm{I}^{[32-35]}$ with details on the synthesis conditions, sample environment and cycling temperature that are important in defining the crystal structure. Neutron diffraction experiments and first-principle calculations in $\mathrm{FAPbI}_{3}$ assign the observed phase transitions to a change from a hexagonal low temperature (Hex-LT) to a hexagonal intermediate temperature (Hex-IT) phase upon heating the sample whereas via thermal quenching the cubic phase can be kinetically trapped down to $8 \mathrm{~K} \cdot{ }^{[32]}$ Refs. [34] and [35] reported a tetragonal phase, $P 4 / \mathrm{mbm}$, via SCXRD at $285 \mathrm{~K}$ and a further transition at $140 \mathrm{~K}^{[35]}$ which adopts a low temperature tetragonal structure. We also note that the hightemperature region of NTE that we observe is correlated with the spectroscopic signature in optical photoluminescence (PL) data where a sudden blue-shift of the PL peak attributed to the lattice expansion $^{[36]}$ is observed at temperatures between 120 and $140 \mathrm{~K} .{ }^{[10,25]}$ Moreover, it has 


\section{WILEY-VCH}

been shown that ferroelectric domains are formed in the lower symmetry phases at lower temperature, ${ }^{[37,38]}$ which most likely cause the NTE below $54.5 \mathrm{~K}$.

The normalized change of the lattice parameter $(\Delta L / L(280 \mathrm{~K}))$ in the $c$-direction, extracted from $T$-dependent SCXRD measurements, is shown in Figure 2(a) and confirms the lattice expansion in the same temperature region. However, the total volume of the lattice (shown in the Supporting Information (Figure S3)) exhibits a steady decrease with decreasing temperature due to a large contraction along the $a$-axis. Data reduction for $T>173 \mathrm{~K}$ has been performed using the RT phase, $P 6_{3} / m m c$, while for $T<173 \mathrm{~K}$ the space group $P 6_{3} / m$ was used. From the PXRD patterns in the proximity of the phase transition, as illustrated in Figure 2(b), a new reflection appears at $170 \mathrm{~K}$ signaling a phase transition (for full temperature range PXRD waterfall plots see Figure S4). The phases above- and below $170 \mathrm{~K}$ were best described by the space groups $P 6_{3} / m m c$ and $P 6_{3} / m$, respectively, using Le Bail refinement. The result of the refinement at 300 and $160 \mathrm{~K}$ can be seen in Figure S5. The new reflection corresponds to the (111) lattice plane in $P 6_{3} / m$ in agreement with the single-crystal precession maps in Figure S6. The evolution of the lattice parameters from PXRD also confirms the NTE in the $c$-direction in the same temperature range, accompanied by a large contraction along the $a$-axis (Figure S7).

Table I: Summary of the structural phase transitions with increasing temperature observed in $\mathrm{FAPbI}_{3}$.

\begin{tabular}{|c|c|c|c|c|c|c|}
\hline \multirow[t]{2}{*}{$\mathrm{FAPbl}_{3}$} & \multirow{2}{*}{$\begin{array}{l}\text { Transition/abrupt } \\
\text { changes in } \Delta L / L\end{array}$} & \multirow[t]{2}{*}{ Phase } & \multicolumn{2}{|c|}{ Lattice parameters } & \multirow{2}{*}{$\begin{array}{l}\text { Space } \\
\text { group }\end{array}$} & \multirow[t]{2}{*}{ Synthesis method } \\
\hline & & & $a(\AA)$ & $c(\AA)$ & & \\
\hline $\begin{array}{l}\text { Ref. [32] } \\
\text { (Neutron } \\
\text { diffraction) }\end{array}$ & $\begin{array}{l}15 \mathrm{~K} \\
220 \mathrm{~K} \\
390 \mathrm{~K} \\
\end{array}$ & $\begin{array}{l}\text { Hexagonal-LT } \\
\text { Hexagonal-IT } \\
\text { Cubic }\end{array}$ & $\begin{array}{l}8.5070 \\
8.6226 \\
6.3855\end{array}$ & $\begin{array}{l}7.9505 \\
7.9458 \\
6.3855\end{array}$ & $\begin{array}{l}\mathrm{P}_{3} / \mathrm{m} \\
\mathrm{P}_{3} / \mathrm{mmc} \\
\mathrm{Pm} 3 \mathrm{~m}\end{array}$ & $\begin{array}{l}\text { Solution } \\
\text { process/precipitation }\end{array}$ \\
\hline $\begin{array}{l}\text { Ref. [33] } \\
\text { (SCXRD) }\end{array}$ & $\begin{array}{l}150 \mathrm{~K} \\
293 \mathrm{~K}\end{array}$ & $\begin{array}{l}\text { Trigonal } \\
\text { Trigonal }\end{array}$ & $\begin{array}{l}17.7914 \\
8.9817\end{array}$ & $\begin{array}{l}10.9016 \\
11.006\end{array}$ & $\begin{array}{l}\text { P3m1 } \\
\text { P3 }\end{array}$ & $\begin{array}{l}\text { Inverse temperature } \\
\text { crystallization }\end{array}$ \\
\hline $\begin{array}{l}\text { Ref. [34] } \\
\text { (PXRD) }\end{array}$ & $285 \mathrm{~K}$ & Tetragonal & 8.92276 & 6.32626 & $\mathrm{P} 4 / \mathrm{mbm}$ & $\begin{array}{l}\text { Solution } \\
\text { process/precipitation }\end{array}$ \\
\hline $\begin{array}{l}\text { Ref. [35] } \\
\text { (SCXRD) }\end{array}$ & $\begin{array}{l}140 \mathrm{~K} \\
285 \mathrm{~K}\end{array}$ & $\begin{array}{l}\text { Tetragonal } \\
\text { Tetragonal }\end{array}$ & $\begin{array}{l}8.87516 \\
8.92276\end{array}$ & $\begin{array}{l}6.27916 \\
6.32626\end{array}$ & $\begin{array}{l}\mathrm{P} 4 / \mathrm{mbm} \\
\mathrm{P} 4 / \mathrm{mbm}\end{array}$ & $\begin{array}{l}\text { Inverse temperature } \\
\text { crystallization }\end{array}$ \\
\hline $\begin{array}{l}\text { present work } \\
\text { (thermal expansion } \\
\text { and SCXRD/PXRD) }\end{array}$ & $\begin{array}{l}54.5 \mathrm{~K} \\
173.0 \mathrm{~K}\end{array}$ & - & $\begin{array}{l}- \\
8.5900 \\
\text { (PXRD } \\
\text { value) }\end{array}$ & $\begin{array}{l}- \\
7.93289 \\
\text { (PXRD } \\
\text { value) }\end{array}$ & $\overline{\mathrm{P}} \mathrm{G}_{3} / \mathrm{m}$ & $\begin{array}{l}\text { Inverse temperature } \\
\text { crystallization }\end{array}$ \\
\hline
\end{tabular}




\section{WILEY-VCH}

Table II: Summary of the structural phase transitions with increasing temperature observed in $\mathrm{FAPbBr}_{3}$.

\begin{tabular}{|c|c|c|c|c|c|c|c|}
\hline \multirow[t]{2}{*}{$\mathrm{FAPbBr}_{3}$} & \multirow{2}{*}{$\begin{array}{l}\text { Transition/ } \\
\text { Abrupt } \\
\text { changes } \\
\Delta L / L\end{array}$} & \multirow[t]{2}{*}{ Phase } & \multicolumn{3}{|c|}{ Lattice parameters } & \multirow{2}{*}{$\begin{array}{l}\text { Space } \\
\text { group }\end{array}$} & \multirow[t]{2}{*}{ Synthesis method } \\
\hline & & & $a(\AA)$ & $b(\AA)$ & $c(\AA)$ & & \\
\hline $\begin{array}{l}\text { Ref. [27] } \\
\text { (PXRD) }\end{array}$ & $\begin{array}{l}100 \mathrm{~K} \\
175 \mathrm{~K} \\
275 \mathrm{~K} \\
\end{array}$ & $\begin{array}{l}\text { Orthorhombic } \\
\text { Tetragonal } \\
\text { Cubic }\end{array}$ & $\begin{array}{l}8.37433 \\
8.41525 \\
5.98618 \\
\end{array}$ & 11.8609 & $\begin{array}{l}8.38073 \\
5.94735\end{array}$ & $\begin{array}{l}\text { Pnma } \\
P 4 / m b m \\
P m-3 m\end{array}$ & $\begin{array}{l}\text { Solution } \\
\text { process/precipitation }\end{array}$ \\
\hline $\begin{array}{l}\text { Ref. [28] } \\
\text { (SCXRD) }\end{array}$ & $\begin{array}{l}100 \mathrm{~K} \\
185 \mathrm{~K} \\
295 \mathrm{~K} \\
\end{array}$ & $\begin{array}{l}\text { Trigonal } \\
\text { Cubic } \\
\text { Cubic }\end{array}$ & $\begin{array}{l}8.3470 \\
5.9582 \\
6.0134 \\
\end{array}$ & $\begin{array}{l}8.3470 \\
5.9582 \\
6.0134\end{array}$ & $\begin{array}{l}10.2180 \\
5.9582 \\
6.0134 \\
\end{array}$ & $\begin{array}{l}R-3 \\
P m-3 m \\
P m-3 m\end{array}$ & $\begin{array}{l}\text { Inverse temperature } \\
\text { crystallization }\end{array}$ \\
\hline $\begin{array}{l}\text { Present work } \\
\text { (thermal } \\
\text { expansion } \\
\text { and SCXRD) }\end{array}$ & $\begin{array}{l}120.5 \mathrm{~K} \\
152.0 \mathrm{~K} \\
170 \mathrm{~K} \\
265.0 \mathrm{~K}\end{array}$ & $\begin{array}{l}\text { Orthorhombic } \\
\text { Tetragonal } \\
\text { Cubic }\end{array}$ & $\begin{array}{l}8.38742 \\
8.441622 \\
5.98730\end{array}$ & 11.8546 & 8.37959 & $\begin{array}{l}\text { Pnma } \\
\text { P4/mbm } \\
\text { Pm-3m }\end{array}$ & $\begin{array}{l}\text { Inverse temperature } \\
\text { crystallization }\end{array}$ \\
\hline
\end{tabular}

The linear thermal expansion for $\mathrm{FAPbBr}_{3}$ is shown in Figure 3(a). In total, three abrupt phase transitions are found at 120.5, 152 and $161.3 \mathrm{~K}$ (Figure 3(b-d)) with far higher resolution than was achieved in recent measurements of the temperature-dependent dielectric constant. ${ }^{[28]}$ While abrupt changes are seen at 120.5 and $161.3 \mathrm{~K}$, the feature at $152 \mathrm{~K}$ has a small shoulder on the low-temperature side as can be seen in $\alpha(T)$. Above $170 \mathrm{~K}, \mathrm{FAPbBr}_{3}$ shows two other features with increasing temperature that are illustrated in figures $3(\mathrm{c}, \mathrm{d})$ : at around $182 \mathrm{~K}$, $\Delta L(T) / L$ exhibits a small region of NTE with decreasing temperature in a global plateau-like structure. At $265 \mathrm{~K}$, another faint feature is visible. Temperature evolution of the PXRD patterns in $\mathrm{FAPbBr}_{3}$ for two selected ranges of temperature has been depicted in Figure 3 (e,f). The new peaks after the first transition at $250 \mathrm{~K}$ were successfully indexed with the space group $P 4 / m b m$ using a pseudocubic tetragonal cell with the unit cell parameters $a=b \approx \sqrt{2} a_{\mathrm{c}}$ and $c \approx a_{c}$ where $a_{c}$ is the lattice constant of the cubic phase. This corresponds to an in-phase octahedral tilt of $\mathrm{a}^{0} \mathrm{a}^{0} \mathrm{c}^{+}$in Glazer's notation. ${ }^{[39]}$ The full temperature range PXRD waterfall plots are represented in Figure S8. New peaks are marked with asterisks indicating the structural changes around the temperatures observed via dilatometry. The corresponding Bragg peak locations at $250 \mathrm{~K}$ and $100 \mathrm{~K}$ are indicated in Figure (3e,f) for $\mathrm{FAPbBr}_{3}$ and $\mathrm{PbBr}_{2}$. To correlate the new peaks that appeared in PXRD, the SCXRD precession maps, as seen in Figure S10, were made. In the precision maps, we identify the appearance of new reflections occurring at 


\section{WILEY-VCH}

$250 \mathrm{~K}$, which indicate phase transitions to a structure with lower symmetry. All reflections including the weak ones were successfully indexed by a pseudocubic tetragonal cell and are in agreement with the systematic absences required by the space group $P 4 / \mathrm{mbm}$. We found that the crystal had undergone twinning during the phase transition where we identified two additional domains rotated $90^{\circ}$ with respect to the first domain, aligning the tetragonal $c$-axes of the two twin domains along the diagonals [110] and [1-10], similar to what is seen in MAbased compounds. ${ }^{[40]}$ Comparing the $d$-spacings in PXRD and SCXRD represented in table SIII reveal a perfect match. The PXRD and SCXRD results are in agreement with the tetragonal phase suggested by Schueller et al. ${ }^{[27]}$ using PXRD, while Govinda et al. ${ }^{[28]}$ found it to maintain the small cubic structure using SCXRD.

At $100 \mathrm{~K}$ we found an orthorhombic unit cell with $a \approx c \approx \sqrt{2} a_{\mathrm{c}}$ and $b \approx 2 a_{\mathrm{c}}$ to give the best description of the superlattice reflection observed in SCXRD. Here the data indicate two twins rotated 120 degrees around the [0-11] and [110] directions. The precession maps in Figure S11 calculated with this cell are in agreement with the space group Pnma. However, this space group does not describe all of the new reflections appearing in the PXRD between $130 \mathrm{~K}$ and $170 \mathrm{~K}$ as shown in Figure 3(f). This points towards a lower symmetry than reported by Schueller et $a l .{ }^{[27]}$ Several of the indexed reflections in PXRD did not show intensity in the SCXRD data. A few of the reflections have not been found in SCXRD, presumably due to the extremely low intensity of some of the superlattice reflections. The comparison between the $d$-spacings of the lattice plane found in both PXRD and SCXRD show a good match, see Table SIV.

To conclude, we have presented an investigation of the linear thermal expansion of bulk single crystals of lead halide perovskites in the temperature range between 4.2 and $280 \mathrm{~K}$ supported by transport and experiments under steady-state illumination for MA-based perovskites. Solid evidence for structural phase transitions superimposed on a smoothly varying background was found for all compounds, though its overall shape depends strongly on the individual material. We observe that FA-based HOIPs exhibit a variety of interesting properties such as two regions 


\section{WILEY-VCH}

of negative thermal expansion $\left(\delta-\mathrm{FAPbI}_{3}\right)$ with a large negative thermal expansion coefficient and a cascade of sharp transitions $\left(\mathrm{FAPbBr}_{3}\right)$ with decreasing temperature. With temperaturedependent PXRD, we have identified the corresponding phases for the FA-based compounds. For $\mathrm{FAPbBr}_{3}$, the symmetry changes identified by X-ray diffraction data analysis are linked to the tilting of the $\mathrm{PbBr}_{6}$ octahedra. These unexpected observations underline the strength of thermal expansion experiments to probe the lattice parameters and to precisely determine the structural phase transition temperatures. Since any structural change has severe consequences on the phononic and electronic structure, the information provided by this study can be employed strategically to predict changes in the optical and charge transport properties that are currently under intense investigation. It is noteworthy that the structural phase transitions for HOIPs of different cations are analogous; meaning most findings of phase transitions can be translated, with generally just a shift in the temperature scale as a function of Goldschmidt's tolerance factors. This information extends across temperatures above room temperature for some other HOIP relatives.

\section{Experimental section}

Device synthesis and characterization All bulk single crystals with flat facets up to $4 \mathrm{x} 4 \mathrm{~mm}^{2}$ and a thickness of around $L=1 \mathrm{~mm}$ were grown using the inverse temperature crystallization (ITC) method, as reported in the literature. ${ }^{[41,42]}$ For all the results mentioned above the freshly synthesized crystals have been used unless indicated. For transport experiments, Au was evaporated on the sample edges either in a four- or six-terminal configuration (in van der Pauw or in Hall/bar geometry) and Au wires with a diameter of $25 \mu \mathrm{m}$ were attached by means of $\mathrm{Ag}$ conductive paste to the sample that had been mounted on a chip carrier. ${ }^{[16]}$

Thermal expansion experiments Thermal expansion measurements were carried out using a high-resolution (down to 0.02 Angstroms) capacitive dilatometer. ${ }^{[19,20]}$ The dilatometer cell is made from a $\mathrm{CuBe}$ alloy and was attached to a specifically designed probe. ${ }^{[20]}$ This probe is 


\section{WILEY-VCH}

put into a vacuum tube and filled with a sufficient amount of ${ }^{4} \mathrm{He}$ contact gas to ensure thermal equilibrium during the measurement. All thermal expansion curves were recorded during the warm up (with a maximum warm up time of $72 \mathrm{~h}$ ) to ensure quasi-adiabatic conditions. The length change $\Delta L$ of the sample along the main axis was measured using an ultrahigh-resolution Andeen-Hagerling capacitance bridge (AH2500). The absolute values of capacitance were always kept between 15 and $25 \mathrm{pF}$.

Magneto-transport experiments A special probe that is equipped with an optical fibre, a calibrated Cernox thermometer and a strain gauge heater was employed to monitor the temperature. The probe was placed in a vacuum tube, equipped with an additional inner vacuum chamber to perform transport experiments from 4.2 to $300 \mathrm{~K}$, in the presence of a small amount of ${ }^{4} \mathrm{He}$ contact gas. The temperature was monitored using a standard Lakeshore temperature controller. The AC measurements were performed using a Keithley 6221 current source at frequencies around $13 \mathrm{~Hz}$ and Stanford 830 Lock-in amplifiers with suitable pre-amplifiers. The applied excitation current varied between $10 \mathrm{nA}$ and $1 \mu \mathrm{A}$ (always in the ohmic regime). A solid-state laser $(373 \mathrm{~nm})$ was utilized for the photoconductivity measurements at a fixed power of $5 \mathrm{~mW} \cdot{ }^{[16]}$

Temperature dependent SCXRD measurements Temperature dependent single-crystal diffraction data were collected on an Oxford Diffraction Supernova diffractometer using a Mo $\mathrm{K} \alpha$ source $(\lambda=0.71073 \AA)$ operated at $50 \mathrm{kV}$ and $0.80 \mathrm{~mA}$ and a detector distance of $73 \mathrm{~mm}$. $\mathrm{FAPbX}_{3}$ single crystals with dimensions $\sim 40 \times 40 \times 10(\mu \mathrm{m})^{3}$ were selected and mounted on a goniometer head using paratone- $\mathrm{N}$ oil. The crystals were cooled between $280-100 \mathrm{~K}$ using an Oxford Cryosystems Cryostream 700 device. Data were collected in steps of $2 \mathrm{~K}$ and the temperature was allowed to equilibrate for 1-2 minutes prior to data collection. The collection strategy used allowed for $100 \%$ completeness of the $\mathrm{Pm}-3 \mathrm{~m}$ space group for $\mathrm{FAPbBr}_{3}$ and $\mathrm{P}_{3} / \mathrm{m}$ for $\mathrm{FAPbI}_{3}$ with an exposure time of $7 \mathrm{~s}$ per frame. The program CrysalisPro32 was used to control the data collection and for the subsequent data reduction. The fitted lattice parameters 


\section{WILEY-VCH}

were averaged in pairs of two in order to reduce errors introduced by small variations in the goniometer head placement at the start of each temperature increment.

Data for precession maps were collected on a Bruker APEX2 diffractometer with an Ag X-ray source $(\lambda=0.56086 \AA)$ operated at $50 \mathrm{kV}$ and $0.88 \mathrm{~mA}$ with a detector distance of $45 \mathrm{~mm}$. The same crystals were used for the temperature scan. Five $\omega$ settings were selected combined with $\varphi$ scans with a $90 \mathrm{~s}$ exposure time per degree for a total of $16 \mathrm{~h}$. The crystals were cooled at 360 $\mathrm{K} / \mathrm{h}$ between each measurement at 280,250 , and $100 \mathrm{~K}$ using an Oxford Cryosystems Cryostream 700 device. Collection and reduction were carried out in the APEX3 software.

Temperature dependent PXRD measurements Capillaries were mounted on the sample spinner at beamline P02.1 (PETRA III, DESY, Hamburg, Germany). ${ }^{[43]}$ An initial diffraction pattern of the sample was collected at room temperature using a PerkinElmer XRD1621 area detector at a wavelength of $0.20684 \AA$. The sample was cooled directly to $100 \mathrm{~K}$, using an Oxford Cryosystems Cryostream 700 device, and then warmed in $10 \mathrm{~K}$ steps to to $300 \mathrm{~K}$, with diffraction patterns collected at each step. Finally, the sample was cooled to $110 \mathrm{~K}$ with diffraction patterns again collected at $10 \mathrm{~K}$ intervals. Diffraction data were calibrated and integrated using the routines available within the DAWN suite. ${ }^{[44]}$ LeBail fits were performed using FullProf. ${ }^{[45]}$

Fluorescence lifetime imaging A $488 \mathrm{~nm}$ pulsed diode laser was employed as excitation source which was set at a repetition rate of $10 \mathrm{MHz}$. The corresponding excitation laser beam was directed into the crystals through an oil immersion objective $(100 \times 1.4 \mathrm{NA})$ of an inverted microscope system. The fluorescence light was collected back and filtered by the main dichroic and cut-off filters. The fluorescence photons were detected by an avalanche photodiode (SPCMAQR-14, Perkin-Elmer). Photon counting and time tagging were performed within a TimeHarp 200 module (PicoQuant), with a time resolution of 29 ps per channel. The FLIM images were analyzed using SymphoTime 32 software (PicoQuant). 


\section{Acknowledgements}

The authors acknowledge financial support from the Research Foundation - Flanders (FWO Grant numbers G.06831.15N, G.oB49.15N, G983.19N and ZW15_09-GOH6316), the Research Foundation - Flanders postdoctoral fellowships to J.A.S., M.K., H.Y. and E.D. (FWO Grant numbers $12 \mathrm{Y} 7218 \mathrm{~N}, 12 \mathrm{Y} 6418 \mathrm{~N}, 12 \mathrm{R} 8718 \mathrm{~N}$ and $12 \mathrm{O} 3719 \mathrm{~N}$, respectively), the KU Leuven Research Fund (C14/15/053), the Flemish government through long term structural funding Methusalem (CASAS2, Meth/15/04), the Hercules foundation (HER/11/14). ) and the EC through the Marie Curie ITN project iSwitch (GA-642196). The support of the HFMLRU/FOM, member of the European Magnetic Field Laboratory (EMFL), is also acknowledged. Martin Ottesen and Martin Bremholm gratefully acknowledge funding from the Danish Council for Independent Research under the Sapere Aude program (7027-00077B). Martin Bremholm also thanks CMC for financial support. CMC is a Center of Excellence funded by the Danish National Research Foundation (DNRF93).

Received: ((will be filled in by the editorial staff))

Revised: ((will be filled in by the editorial staff)) Published online: ((will be filled in by the editorial staff)) 


\section{WILEY-VCH}

References

[1] M. A. Green, A. Ho-Baillie \& H. J. Snaith, Nat. Photon. 2014, 8, 506.

[2] N. J. Jeon, J. H. Noh, W. Seok Yang, Y. C. Kim, S. Ryu, J. Seo \& S. Il Seok, Nature 2015, 476, 517.

[3] M. A. Green, K. Emery, Y. Hishikawa, W. Warta \& E. D. Dunlop, Prog. Photovolt. Res. Appl. 2015, 1, 23.

[4] National Renewable Energy Laboratory (NREL), 2018 (accessed July 2018).

[5] V. D’Innocenzo, G. Grancini, M. J. P. Alcocer, A. R. S. Kandada, S. D. Stranks, M. M. Lee, G. Lanzani, H. J. Snaith \& A. Petrozza, Nat. Commun. 2014, 5, 3586.

[6] L. M. Herz, Annu. Rev. Phys. Chem. 2016, 67, 040215.

[7] M. M Lee, J. Teuscher, T. Miyasaka, T. N. Murakami, \& H.J. Snaith, Science 2012, 338, 643-647.

[8] S. D. Stranks, G. E. Eperon, G. Grancini, C. Menelaou, M. J. P. Alcocer, T. Leijtens, L. M. Herz, A. Petrozza, H. J. Snaith, Science 2013, 342, 341.

[9] Y. Chen, H. T. Yi, X. Wu, R. Haroldson, Y. N. Gartstein, Y. I. Rodionov, K. S. Tikhonov, A. Zakhidov, X. -Y. Zhu \& V. Podzorov, Nat Commun 2016, 7, 12253.

[10] H.-H. Fang, R. Raissa, M. Abdu-Aguye, S. Adjokatse, G. R. Blake, J. Even, M. A. Loi, Adv. Funct. Mater. 2016, 25, 2378.

[11] T. Zhao, W. Shi, J. Xi, D. Wang \& Z. Shuai, Scientific Reports 2016, 6, 19968.

[12] A. Miyata, A. Mitioglu, P. Plochocka, O. Portugall, J. Tse-Wei Wang, S. D. Stranks, H. J. Snaith, R. J. Nicholas, Nat. Phys. 2015, 11, 582-587.

[13] K. Galkowski, A. Mitioglu, A. Miyata, P. Plochocka, O. Portugall, G. E. Eperon, J. Tse-Wei Wang, T. Stergiopoulos, S. D. Stranks, H. J. Snaith and R. J. Nicholas, Energy Environ. Sci., 2016, 9, 962-970.

[14] Z. Yang, A. Surrente, K. Galkowski, N. Bruyant, D. K. Maude, A. Abbas Haghighirad, H. J. Snaith, P. Plochocka, and R. J. Nicholas, J. Phys. Chem. Lett., 2017, 8 (8), 1851-1855.

[15] H. T. Yi, X. Wu, X. Zhu, and V. Podzorov, Adv. Mater. 2016, 28, 6509-6514.

[16] M. Keshavarz, S. Wiedmann, H. Yuan, E. Debroye, M. Roeffaers and J. Hofkens, ACS Energy Lett., 2018, 3 (1), 39-45

[17] A. Poglitsch, \& D. J. Weber, Chem. Phys. 1987, 87, 6373.

[18] N. Onoda-Yamamuro, T. Matsuo and H. Suga, J. Phys Chem. Solids 1990, 51, No. 12., 1383-1395.

[19] R. Küchler, T. Bauer, M. Brando, and F. Steglich, Rev. Sci. Instrum. 2012, 83, 095102.

[20] R. Küchler, A. Wörl, P. Gegenwart, M. Berben, B. Bryant, and S. Wiedmann, Rev. Sci. Instrum. 2017, 88, 083903.

[21] M. T. Weller, O. J. Weber, P. F. Henry, A. M. Di Pumpoa and T. C. Hansen, Chem. Commun., 2015, 51, 4180.

[22] F. Brivio, J. M. Frost, J. M. Skelton, A. J. Jackson, O. J. Weber, M. T. Weller, A. R. Goni, A. M. A. Leguy, P. R. F. Barnes, and A. Walsh, Phys. Rev. B 2015, 92, 144308.

[23] P. S. Whitfield, N. Herron, W. E. Guise, K. Page, Y. Q. Cheng, I. Milas \& M. K. Crawford, Scientific Reports 2016, 6, 35685.

[24] C. Ge, Mingyu Hu, P. Wu, Q. Tan, Z. Chen, Y. Wang, J. Shi, and J. Feng, J. Phys. Chem. C 2018, 122 (28), pp 15973-15978.

[25] A. D. Wright, C. Verdi, R. L. Milot, G. E. Eperon, M. A. Pe'rez-Osorio, H. J. Snaith, F. Giustino, M. B. Johnston \& L. M. Herz, Nature Communications 2016, 7, 11755.

[26] K. L. Brown, S. F. Parker, I. Robles García, S. Mukhopadhyay, V. García Sakai2 and C. Stock, Phys. Rev. B 2017, 96, 174111.

[27] E. C. Schueller, G. Laurita , D. H. Fabini, C. C. Stoumpos, M. G. Kanatzidis, and R. Seshadri, Inorg. Chem., 2018, 57 (2), 695-701 


\section{WILEY-VCH}

[28] S. Govinda, B. P. Kore, D. Swain, A. Hossain, C. De, T. N. Guru Row, and D. D. Sarma, J. Phys. Chem. C 2018, 122, 13758-13766.

[29] K. Takenaka, Sci. Technol. Adv. Mater. 2012, 13, 013001.

[30] K. Takenaka, and H. Takagi, Appl. Phys. Lett. 2005, 87, 261902.

[31] K.Takenaka, Y. Okamoto, T. Shinoda, N. Katayama \& Y. Sakai, Nature Communications 2017, 8, 14102.

[32] T. Chen, B. J. Foley, C. Park, C. M. Brown, L. W. Harriger, J. Lee, J. Ruff, M. Yoon, J.J. Choi, Seung-Hun Lee, Science Advances, 2016, 160, 1650.

[33] C. C. Stoumpos, C. D. Malliakas, M. G. Kanatzidis, Inorg. Chem. 2013, 52 (15), 9019-9038.

[34] D. H. Fabini, C. C. Stoumpos, G. Laurita, A. Kaltzoglou, A. G. Kontos, P. Falaras, M. G. Kanatzidis, R. Seshadri, Angew. Chem., Int. Ed. 2016, 55 (49), 15392-15396.

[35] S. Sun, Z. Deng, Y. Wu, F. Wei, F. Halis Isikgor, F. Brivio, M. W. Gaultois, J. Ouyang, P. D. Bristowe, A. K. Cheetham, G. Kieslich, T. B. Bein, H. J. Snaith, R. H. Friend, H.-K. Mao, Chem. Commun. 2017, 53 (54), 7537-7540.

[36] D. H. Fabini, G. Laurita, J.S.Bechtel, C. C. Stoumpos, H.A.Evans, A.G.Kontos, Y.S.Raptis, P.Falaras, A.Van der Ven, M. G. Kanatzidis,R.Seshadri, J. Am. Chem. Soc. 2016, 138, 11820-11832.

[37] Y. Kutes, L. Ye, Y. Zhou, S. Pang, B. D. Huey, and N. P. Padture, J Phys Chem Lett 2014, 5, 3335-3339.

[38] Y. Rakita, O. Bar-Elli, E. Meirzadeh, H. Kaslasi, Y. Peleg, G. Hodes, I. Lubomirsky, D. Oron, D. Ehre, and D. Cahen, PNAS 2017, 114 (28) E5504-E5512.

[39] A. M. Glazer, Acta Crystallogr., Sect. B: Struct. Crystallogr. Cryst. Chem. 1972, 28, 3384-3392.

[40] M. Szafrański and A. Katrusiak, The Journal of Physical Chemistry Letters, 2017, 8 (11), 2496-2506.

[41] M. I. Saidaminov, A. L. Abdelhady, B. Murali, E. Alarousu, V. M. Burlakov, W. Peng, I. Dursun, L. Wang, Y. He, G. Maculan, A. Goriely, T. Wu, O. F. Mohammed, \& O. M. Bakr, Nature Communications 2015, 6, 7586 .

[42] A. A. Zhumekenov, M. I. Saidaminov, M. Azimul Haque, E. Alarousu, S. Phukan Sarmah, B. Murali, I. Dursun, X. -H. Miao, A. L. Abdelhady, T. Wu, O. F. Mohammed, and O. M. Bakr, ACS Energy Lett., 2016, 1 (1), pp 32-37.

[43] A.-C. Dippel, H.-P. Liermann, J. T. Delitz, P. Walter, H. Schulte-Schrepping, O. H. Seeck and H. Franz, J. Synchrotron Radiat., 2015, 22, 675-687.

[44] J. Filik, A. W. Ashton, P. C. Y. Chang, P. A. Chater, S. J. Day, M. Drakopoulos, M. W. Gerring, M. L. Hart, O. V. Magdysyuk, S. Michalik, A. Smith, C. C. Tang, N. J. Terrill, M. T. Wharmby and H. Wilhelm, J. Appl. Cryst., 2017, 50, 959-966.

[45] J. Rodrigues-Carvajal, Comm. Powder Diffr. IUCr Newsl., 2001, 26, 12-19. 


\section{WILEY-VCH}

Figures
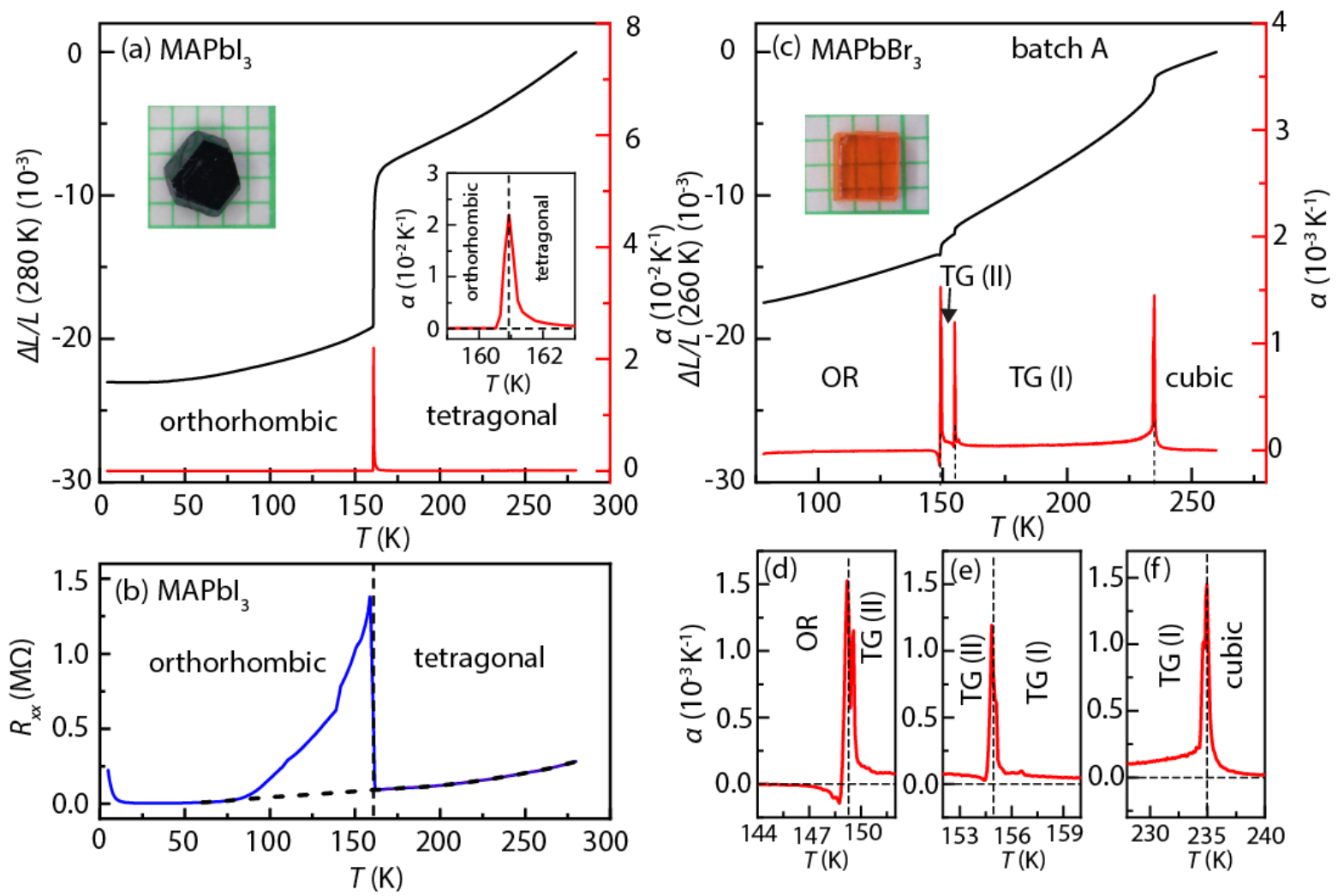

Figure 1. Observation of the structural phase transitions in $\mathrm{MAPbI}_{3}$ and $\mathrm{MAPbBr}_{3}$. (a) $\Delta L / L$ for $\mathrm{MAPbI}_{3}$ and linear thermal expansion coefficient $\alpha$ as a function of temperature. The phase transition from an orthorhombic to a tetragonal phase is found at $160.9 \mathrm{~K}$, see inset for $\alpha(T)$. (b) Longitudinal resistance $R_{x x}$ under steady-state illumination as a function of temperature. The horizontal dashed line indicates the temperature dependence of $R_{x x}$ in the absence of the structural phase transition. (c) $\Delta L / L$ and $\alpha$ as a function of temperature for MAPbBr3 (batch A). $(\mathrm{d}, \mathrm{e}, \mathrm{f})$ Zoom of the thermal expansion coefficient $\alpha$ in the vicinity of the tetragonal phases, see Table S1. OR: orthorhombic, TG (I): tetragonal-I and TG (II): tetragonal-II. 


\section{WILEY-VCH}
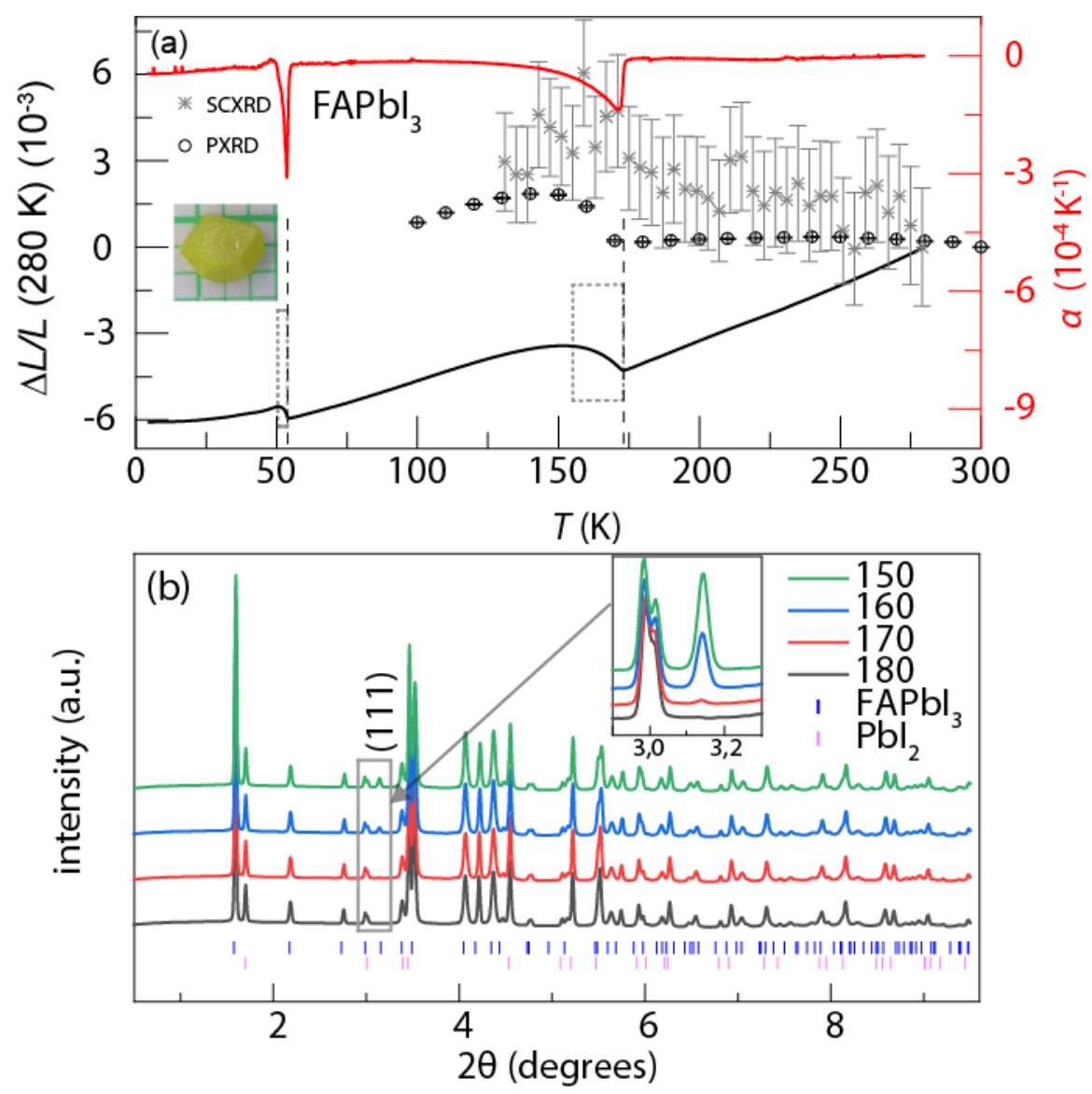

Figure 2. Structural phase transitions and X-Ray diffraction pattern in $\delta-\mathrm{FAPbI}_{3}$. (a) $\Delta L / L$, lattice parameter $c$ (open circles from PXRD, stars from SCXRD) and thermal expansion coefficient $\alpha$ as a function of temperature for $\delta-\mathrm{FAPbI}_{3}$. Two regions of negative thermal expansion are observed below 173 and $54.5 \mathrm{~K}$ highlighted by the dashed boxes. (b) X-Ray diffraction pattern and corresponding Bragg peak locations at $160 \mathrm{~K}$ for $\delta$ - $\mathrm{FAPbI}_{3}$ (dark blue) and $\mathrm{PbI}_{2}$ (pink) extracted from PXRD measurements. 


\section{WILEY-VCH}

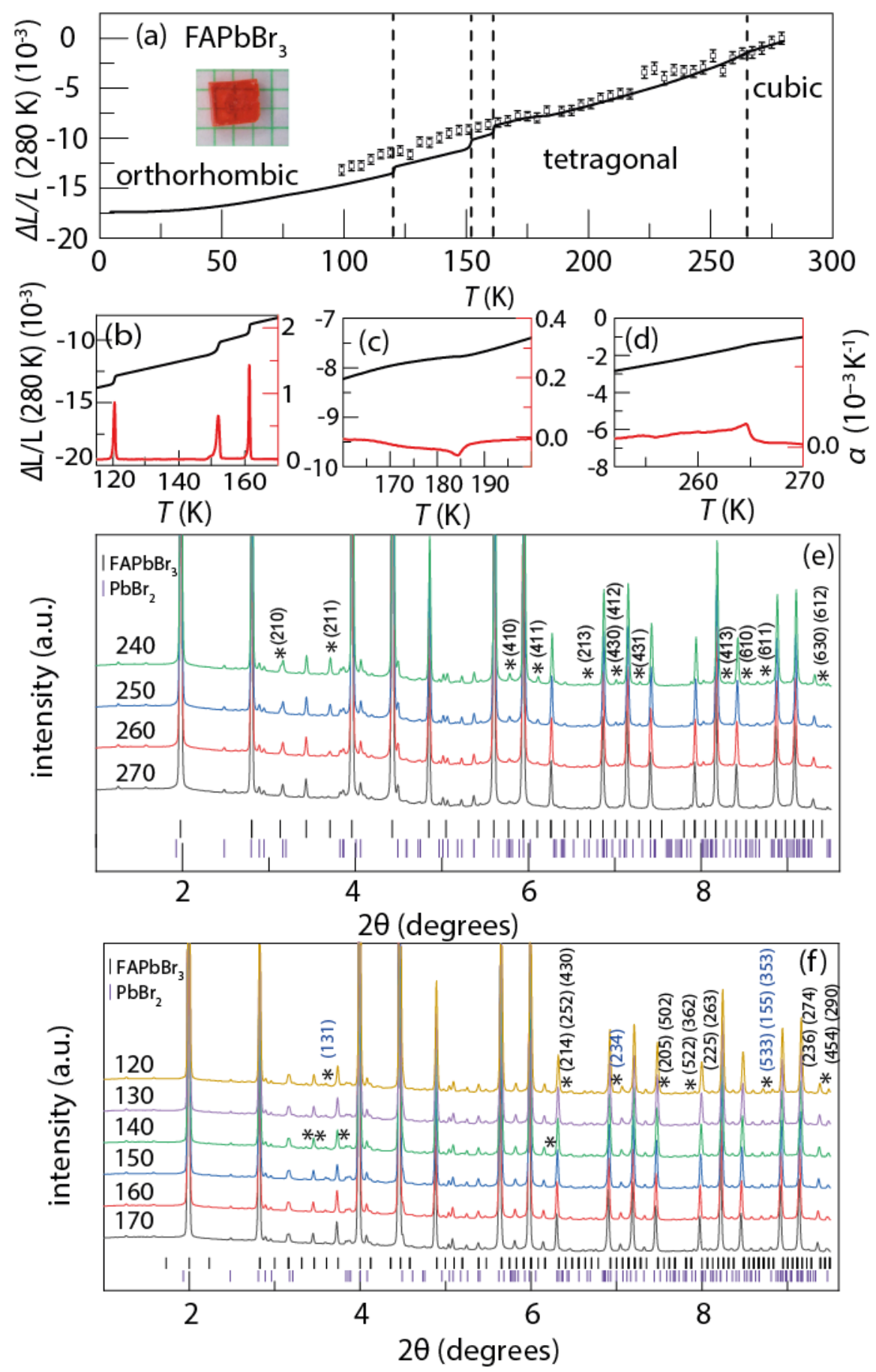

Figure 3. Structural phase transitions and X-Ray diffraction pattern in $\mathrm{FAPbBr}_{3}$. (a) $\Delta L / L$ and lattice parameter $a$ (open circles) as a function of temperature: in $\Delta L / L$, a cascade of sharp phase transition between 120 and $165 \mathrm{~K}$ and a continuously increasing background is observed with increasing temperature. The dashed lines indicate strong changes in $\Delta L / L$. (b,c,d) $\Delta L / L$ and $\alpha$ in different ranges of temperature. (e,f) Powder X-ray diffraction pattern in the high (e) and low (f) temperature range. New peaks are marked with asterisks. The corresponding Bragg peak locations at $250 \mathrm{~K}$ and $100 \mathrm{~K}$ are indicated in (e) and (f) for $\mathrm{FAPbBr}_{3}$ and $\mathrm{PbBr}_{2}$. 


\section{WILEY-VCH}

ToC figure ((Please choose one size: $55 \mathrm{~mm}$ broad $\times 50 \mathrm{~mm}$ high or $110 \mathrm{~mm}$ broad $\times 20 \mathrm{~mm}$ high. Please do not use any other dimensions))

TOC text:

To determine the exact phase transitions temperature of lead-halide perovskite solar cell materials, thermal expansion using a capacitive dilatometer is employed. This strategy led to the discovery of unexplored structural characteristics in the FA-based compounds providing a platform to predict changes in the optical and charge transport properties relevant for applications.

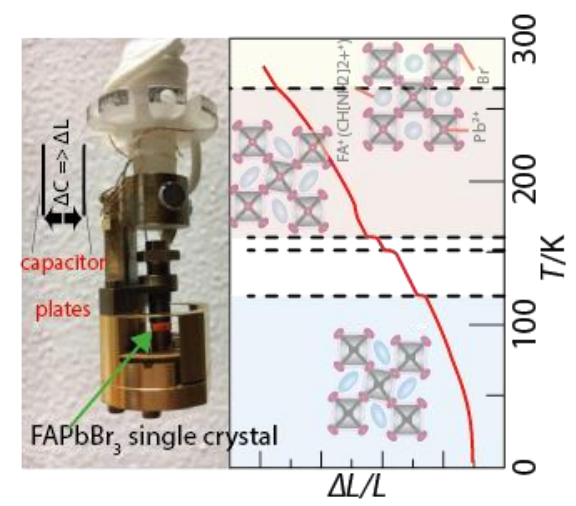




\section{WILEY-VCH}

Copyright WILEY-VCH Verlag GmbH \& Co. KGaA, 69469 Weinheim, Germany, 2018.

\section{Supporting Information}

Tracking Structural Phase Transitions in Lead Halide Perovskites by means of Thermal Expansion

Masoumeh Keshavarz*, Martin Ottesen, Steffen Wiedmann, Michael Wharmby, Robert Küchler, Haifeng Yuan, Elke Debroye, Julian Steele, Johan Martens, Nigel Hussey, Martin Bremholm, Maarten Roeffaers and Johan Hofkens*

Dr. Masoumeh Keshavarz, Dr. Haifeng Yuan, Dr. Elke Debroye and Prof. Johan Hofkens, Molecular Imaging and Photonics, Department of Chemistry, Katholieke Universiteit Leuven, Celestijnenlaan 200F, 3001 Leuven, Belgium.

Martin Ottesen and Dr. Martin Bremholm, Department of Chemistry and iNANO, Aarhus University, Langelandsgade 140, 8000 Aarhus C, Denmark.

Dr. Steffen Wiedmann and Prof. Nigel Hussey, High Field Magnet Laboratory and Institute for Molecules and Materials, Radboud University, Toernooiveld 7, 6525 ED Nijmegen, Netherlands.

Dr. Michael Wharmby, Deutsches Elektronen-Synchrotron (DESY), Notkestr. 85, 22607 Hamburg, Germany.

Dr. Robert Küchler, Max Planck Institute for Chemical Physics of Solids, Nöthnitzer Str. 40, 01187 Dresden, Germany.

Dr. Julian Steele, Prof. Johan Martens and Prof. Maarten Roeffaers, Department of Microbial and Molecular Systems, Centre for Surface Chemistry and Catalysis (COK), KU Leuven, Celestijnenlaan 200F, 3001 Leuven, Belgium.

E-mail: masoumeh.keshavarz@kuleuven.be and johan.hofkens@kuleuven.be 


\section{Charge transport properties under steady-state illumination and thermal expansion for}

\section{MAPbBr3 (batch B)}

In this section, we provide additional insights into thermal expansion experiments using capacitive dilatometry, and, in particular, on the transport experiments under steady-state illumination. An example for thermal expansion $\Delta L(T) / L$ and change carrier transport as a function of temperature for $\mathrm{MAPbBr}_{3}$ (batch $\mathrm{B}$, see below) is illustrated in Figure $\mathrm{S} 1$ (a) and (b), respectively. The longitudinal resistance $R_{x x}(T)$, measured on a sample in van der Pauw geometry, follows the trend found in $\Delta L(T) / L$. The charge carrier concentrations $n$ has been extracted from the slope of a linear Hall effect $\left(R_{x y}\right)$ above and below the phase transition. We found that the carrier concentration increases by $20 \%$ from the orthorhombic to the tetragonal phase.

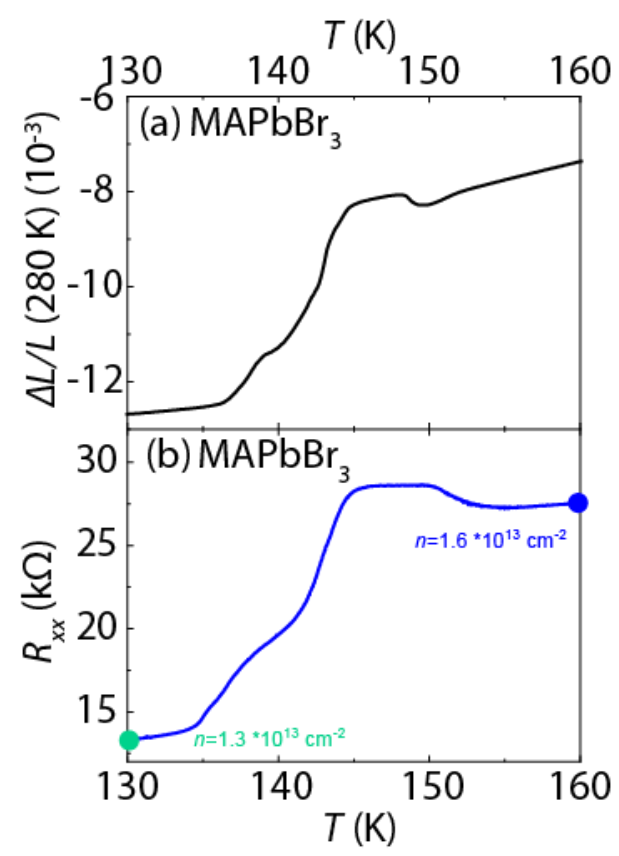

Figure S1. (a) $\Delta L / L$ as a function of temperature measured by capacitive dilatometry for $\mathrm{MAPbBr}$. (b) Longitudinal resistance $R_{x x}$ as a function of temperature measured by transport under steady-state illumination. 
Table SI: Structural phase transitions observed in the temperature range between 4.2 and 300 $\mathrm{K}$ for $\mathrm{MAPbI}_{3}$ and $\mathrm{MAPbBr}_{3}-\mathrm{OR}$ : orthorhombic, TG: tetragonal, C: cubic.

\begin{tabular}{lllll}
\hline Material & Transition & $\begin{array}{l}\text { T }(\mathrm{K}) \\
\text { Ref. [17] }\end{array}$ & $\begin{array}{l}\text { T }(\mathrm{K}) \\
\text { Ref. [18] }\end{array}$ & $\begin{array}{l}\mathrm{T}(\mathrm{K}) \\
\text { present work }\end{array}$ \\
\hline $\mathrm{MAPbl}_{3}$ & $\mathrm{OR} \rightarrow \mathrm{TG}$ & 162.2 & 161.4 & 160.9 \\
\hline $\mathrm{MAPbBr}_{3}$ & $\mathrm{OR} \rightarrow \mathrm{TG}(\mathrm{II})$ & 145.0 & 148.8 & 149.3 \\
& $\mathrm{TG}(\mathrm{II}) \rightarrow \mathrm{TG}(\mathrm{I})$ & 155.1 & 154.4 & 154.9 \\
& $\mathrm{TG}(\mathrm{I}) \rightarrow \mathrm{C}$ & 236.9 & 236.3 & 235.0 \\
\hline
\end{tabular}

We note that the resistivity $\rho_{x x}$, derived from $R_{x x}$ in the absence of a magnetic field is used to calculate the charge carrier mobility together with the charge carrier concentration obtained, e.g. form the Hall resistance. Therefore, even though the carrier concentration might slightly vary, the high resistance value will lead to a significant reduction in carrier mobility below the phase transitions. As a consequence, the identification of the underlying scattering mechanism should be performed in one phase.

\section{Crystal quality effect on the structural phase transitions}

Next, we show the experimental results on time-dependent photoluminescence (PL) on batches $\mathrm{A}$ and $\mathrm{B}$, and the linear thermal expansion on $\mathrm{MAPbBr}_{3}$ (batch $\mathrm{B}$ ), illustrated in Figure $\mathrm{S} 2$. Crystals of batch B were grown one year before the measurements while crystals of batch A were freshly prepared. Between both crystal batches, small variations in precursor solution stoichiometry exist resulting from a continuous refinement of the synthetic procedures. As reported also in recent studies, ${ }^{[\mathrm{S} 1]}$ small variations in precursor solution stoichiometry can affect the crystallinity and increase the number of defects in the perovskite materials. This quality difference between crystals of batch A and B is likely to arise from an unintentional fractional variation in the precursor solution stoichiometry as well as differences in age. The quality difference between these two batches is evident in their optical transmission image in which batch B is more opaque compared to batch A (inset to Figure S2a). As a quality check of the crystals batch A and B, we performed FLIM measurements and observed in general longer photoluminescence (PL) lifetimes for the crystals of batch A compared to batch B which is indeed an indication of lower non-radiative recombination centers, hence lower defect 


\section{WILEY-VCH}

concentrations. ${ }^{[\mathrm{S} 2]}$ To extract the decay times, we fitted the data with a stretched exponential and an exponential using the following formula:

$$
f(t)=A e^{\left(-t / \tau_{1}\right)^{\beta}}+B e^{\left(-t / \tau_{2}\right)} \quad \text { Equation (1) }
$$

where $0<\beta \leq 1$ and $\tau_{1}$ and $\tau_{2}$ are the decay times. Normally, a smaller value of $\beta$ corresponds to a wider distribution of decay times, suggesting a more disordered system. ${ }^{[\mathrm{S} 3]}$ Here $\beta$ for batch $\mathrm{B}\left(\beta_{B}=0.35\right)$ is considerably smaller than that of batch $\mathrm{A}\left(\beta_{A}=0.78\right)$ indicating more disorder in the crystals of batch B compared to A.

In Figure S2(b) we illustrate changes in $\Delta L(T) / L$ in the regions where the crystal structure changes from the orthorhombic to the tetragonal phases (i.e. between 135 and $155 \mathrm{~K}$ ) for batch B. Compared to batch $\mathrm{A}$, the changes in $\Delta L / L$ are rather broad. This is explicitly shown in the linear thermal expansion coefficient that exhibits two clear distinct phases for batch A whereas for batch $\mathrm{B}, \alpha(T)$ contains several maxima between 135 and $155 \mathrm{~K}$ in addition to a region of negative thermal expansion around $148 \mathrm{~K}$, see Figures (c-e). Moreover, the phase transition from tetragonal-I to cubic around $235 \mathrm{~K}$ is absent, instead, $\alpha(\mathrm{T})$ exhibits a maximum around $194 \mathrm{~K}$. 

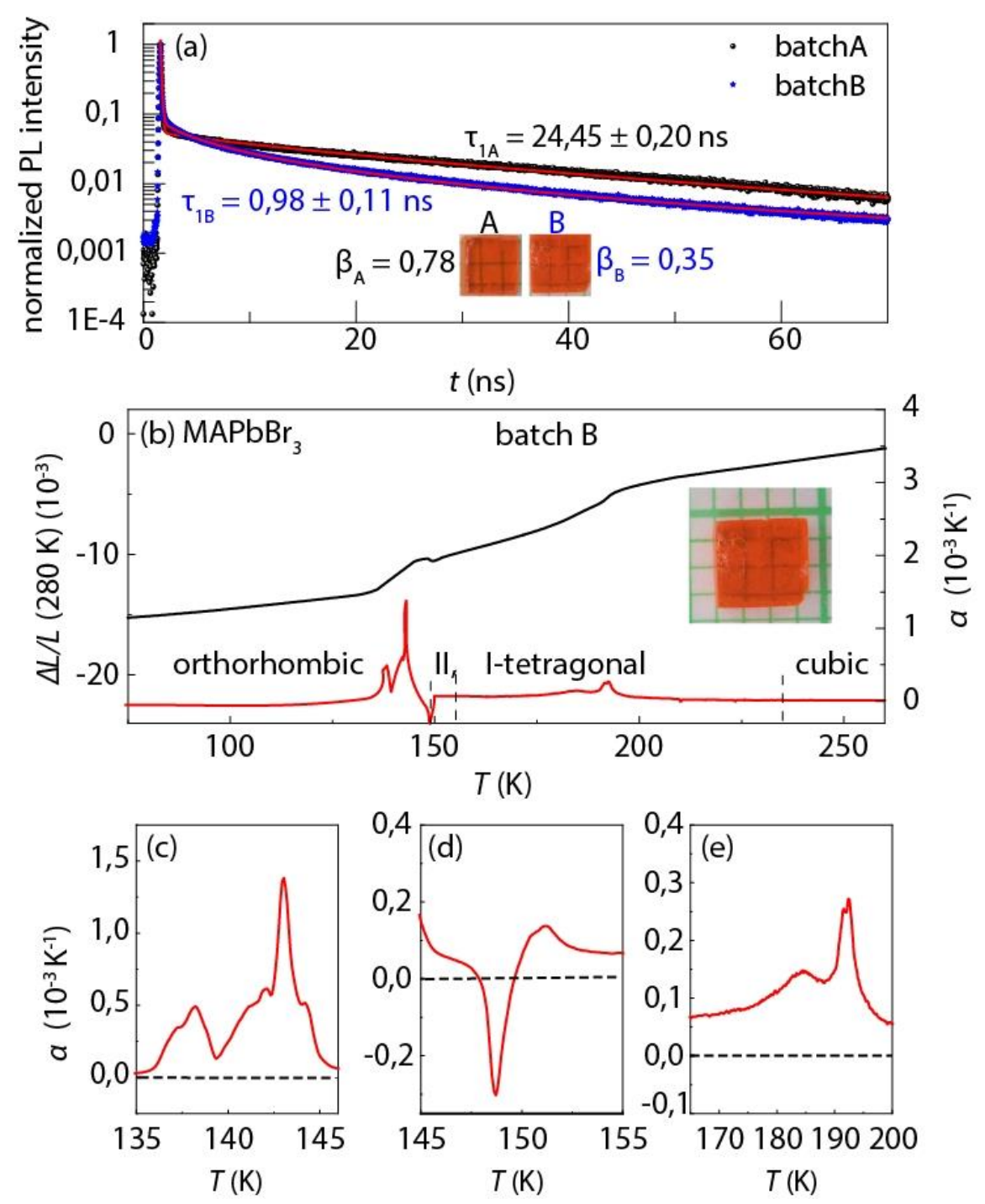

Figure S2. (a) Normalized PL intensity as a function of time for batches A and B. (b) $\Delta L / L$ and $\alpha$ as a function of temperature measured via dilatometry for $\mathrm{MAPbBr}_{3}$ (batch $\mathrm{B}$ ). The dashed vertical lines indicate where the phase transition occurred in batch A. (c-e) Thermal expansion coefficient $\alpha$ as a function of temperature in regions in which we observe changes in $\Delta L / L$. 


\section{WILEY-VCH}

\section{3. $\mathrm{FAPbI}_{3}$ - Supporting information}

A note on the phases of $\mathrm{FAPbI}_{3}$ : This material has in general a low temperature yellow phase and a high temperature black perovskite phase known as $\delta$ and $\alpha$ phase, respectively, in this field's nomenclature. The information provided in Table I include both $\delta$ and $\alpha$ phases while in our study, we only focus on the low temperature yellow phase $\delta$.

\section{Temperature-dependent SCXRD}

The single-crystal diffraction data was reduced using the space groups $P 6_{3} / m m c$ and $P 6_{3} / m$ corresponding to the RT phase and the intermediate phase, respectively. The unit cell parameters, as well as the normalized cell parameters, as a function of temperature can be seen in Figure S3 (a) and (b) respectively. The same trend as the PXRD data has been observed; an increase in the $c$-axis and a decrease in the $a$-axis giving rise to a decrease in the unit cell volume.
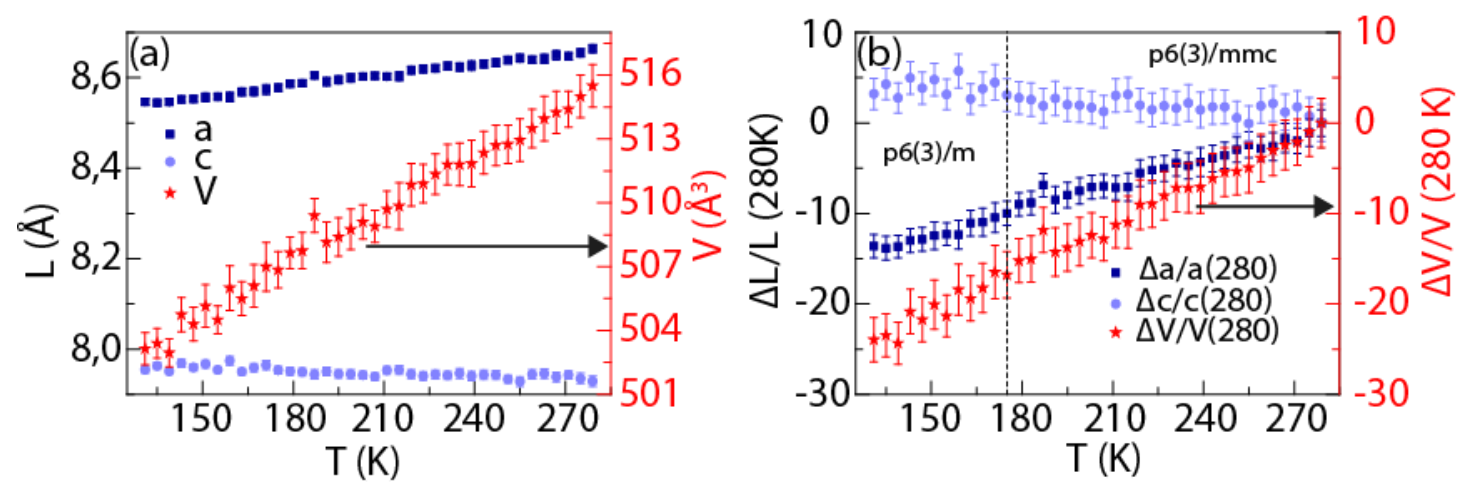

Figure S3. The temperature evolution of the unit cell parameters (a) and the normalized cell parameters (b) extracted from SCXRD measurements. 


\section{WILEY-VCH}

\section{Temperature-dependent PXRD}

The complete PXRD data series can be seen in Figure S4 (full waterfall plot).

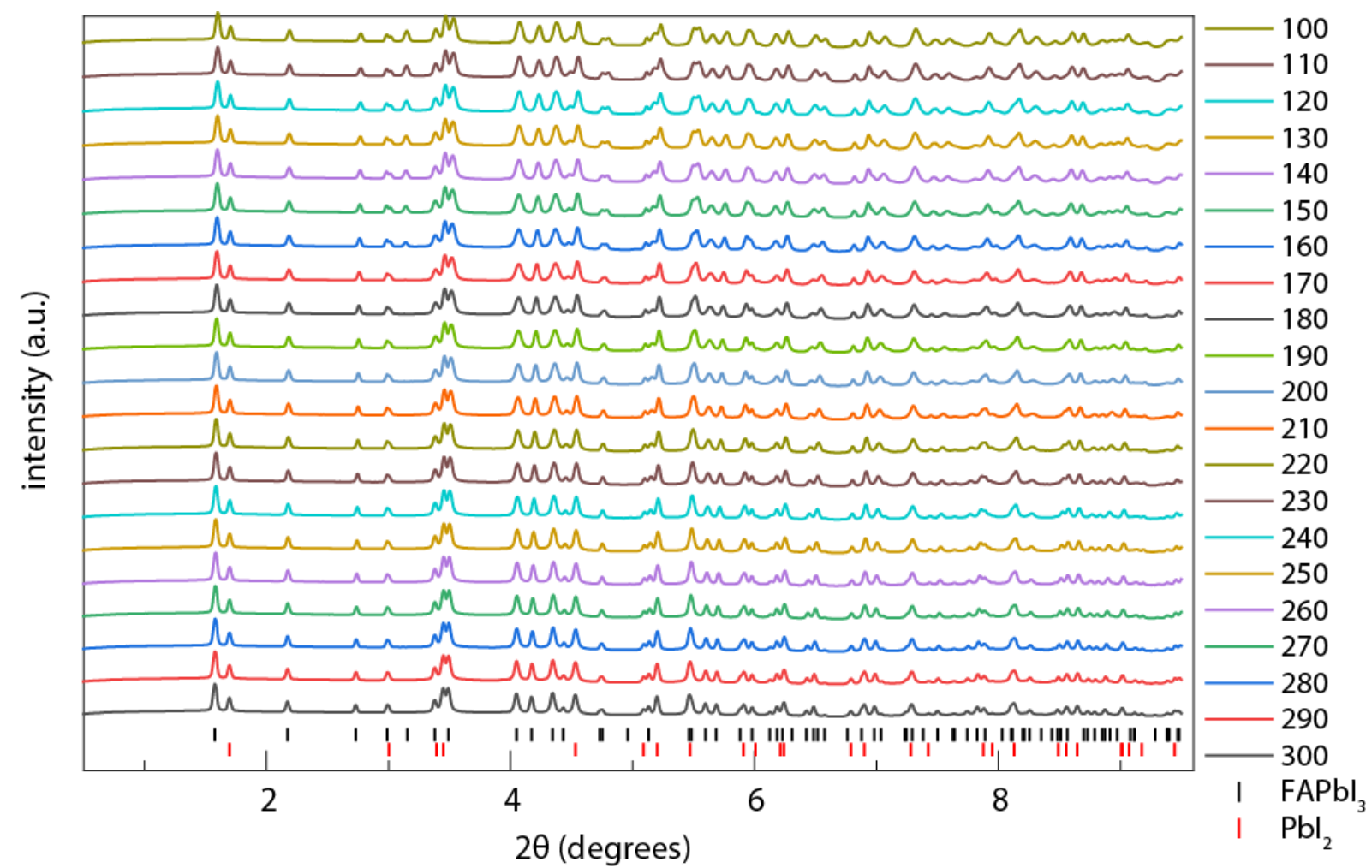

Figure S4. Full temperature range waterfall X-Ray diffraction pattern and corresponding Bragg peak locations at $300 \mathrm{~K}$ for $\delta-\mathrm{FAPbI}_{3}$ (black) and $\mathrm{PbI}_{2}$ (red) extracted from $\mathrm{PXRD}$ measurements.
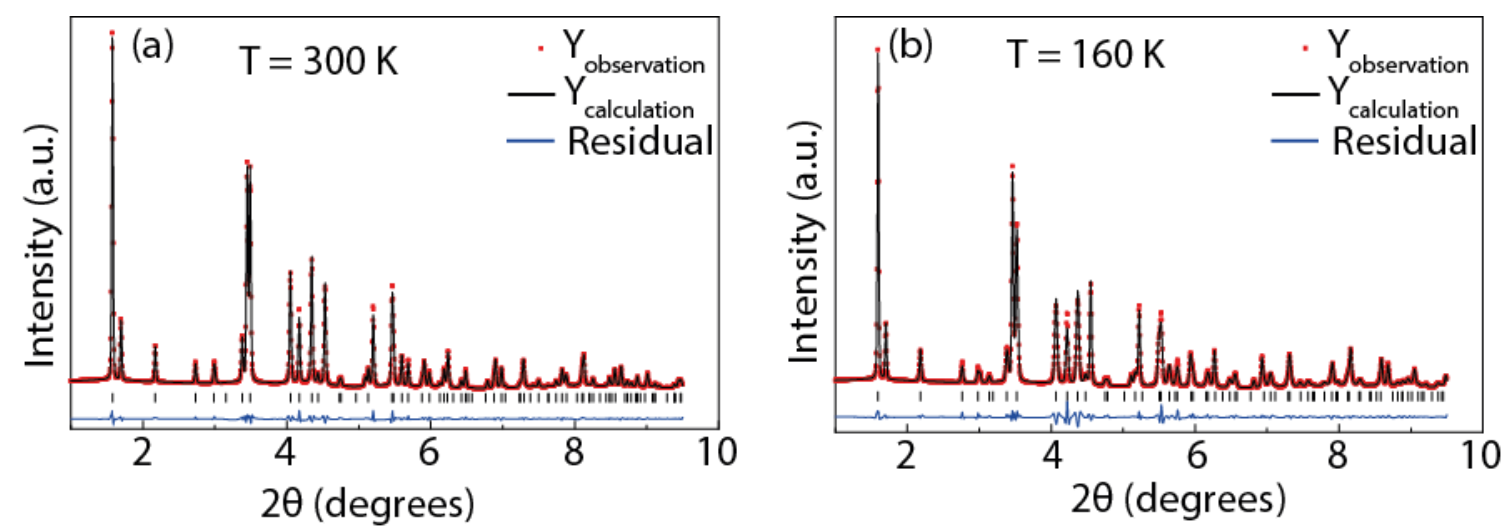

Figure S5: Le Bail refinement with space group $P 6_{3} / m m c$ at $300 \mathrm{~K}$ (left) and $P 6_{3} / m$ at $160 \mathrm{~K}$ (right). Unlabeled peaks are described by the presence of $\mathrm{PbI}_{2}$.

In Figure S5 the Le Bail refinement of the PXRD data for the two phases is shown. The best fit at $300 \mathrm{~K}$ was obtained with the space group $P 6_{3} / m m c$ while the best fit at $160 \mathrm{~K}$ was with $P 6_{3} / m$. This is in agreement with the previously reported 


\section{WILEY-VCH}

phases $^{[\mathrm{S} 4]}$ and with precession maps as seen in Figure S6. Comparing the hk1 plane at $190 \mathrm{~K}$ and $160 \mathrm{~K}$, new reflections are appearing such as the $\{111\}$ symmetry related reflections. This is a result of systematic absences due to the $c$-glide, giving rise to the condition that for indices $h h l: l=2 n$. As the symmetry of the system decreases at the phase transition, the $c$-glide is no longer present, and the previously absent reflections are now allowed. Likewise, it can be seen that the (001) reflection is missing both before and after the phase transition, indicating that the $6_{3}$ screw axis is maintained through the phase transition. Only the space groups $P 6_{3} / m, P 6_{3} 22$, and $\mathrm{PG}_{3}$ fulfill these systematic absences but the former has the better description of the data.

The extracted lattice parameters from the Le Bail refinement can be seen in Figure S7. The large expansion along the $c$-direction between 170 and $130 \mathrm{~K}$ is in excellent agreement with the dilatometry measurements. The $a$-axis experiences a large contraction with decreasing temperature and a small dip after the phase transition, resulting in the unit cell volume steadily decreasing in the entire temperature range.
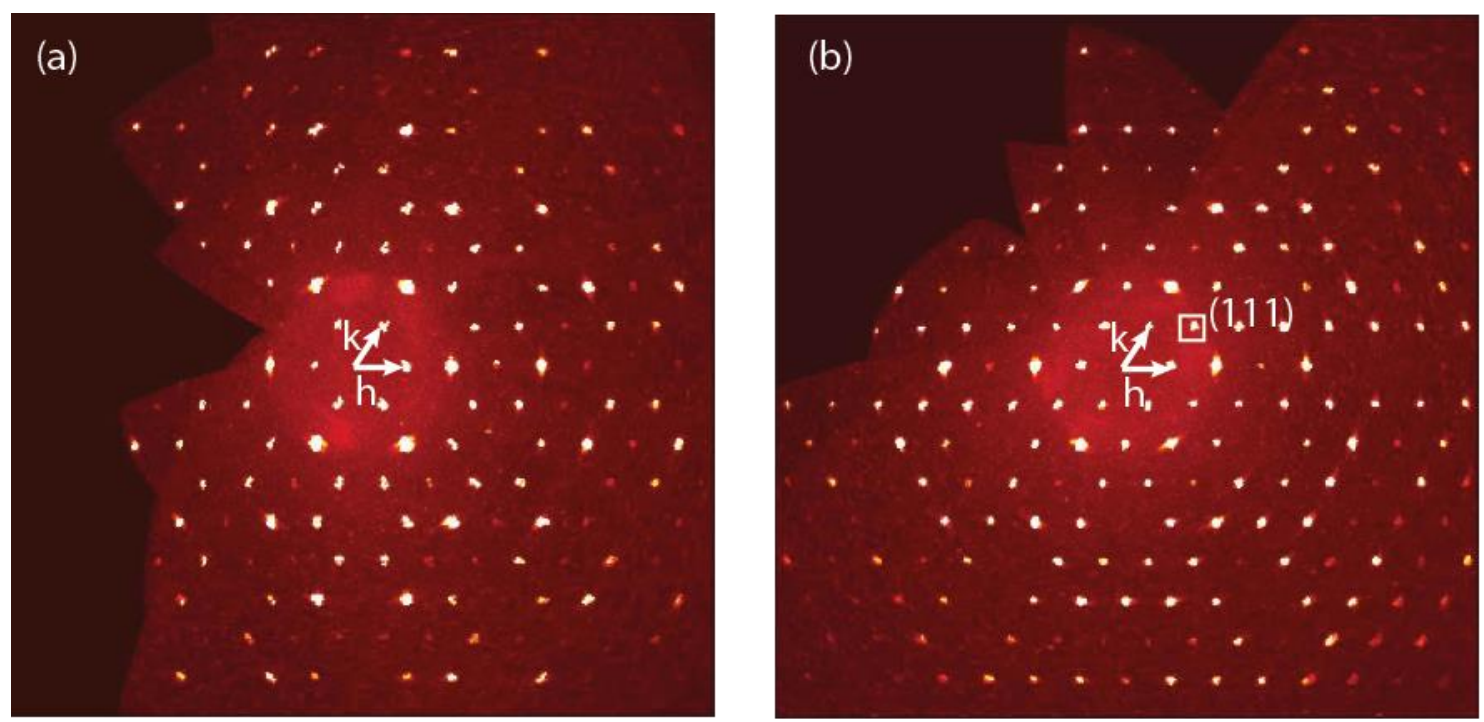

Figure S6: Precession maps of $\mathrm{FAPbI}_{3}$ through the hk1 plane at (a) $190 \mathrm{~K}$ and (b) 160 K. 


\section{WILEY-VCH}
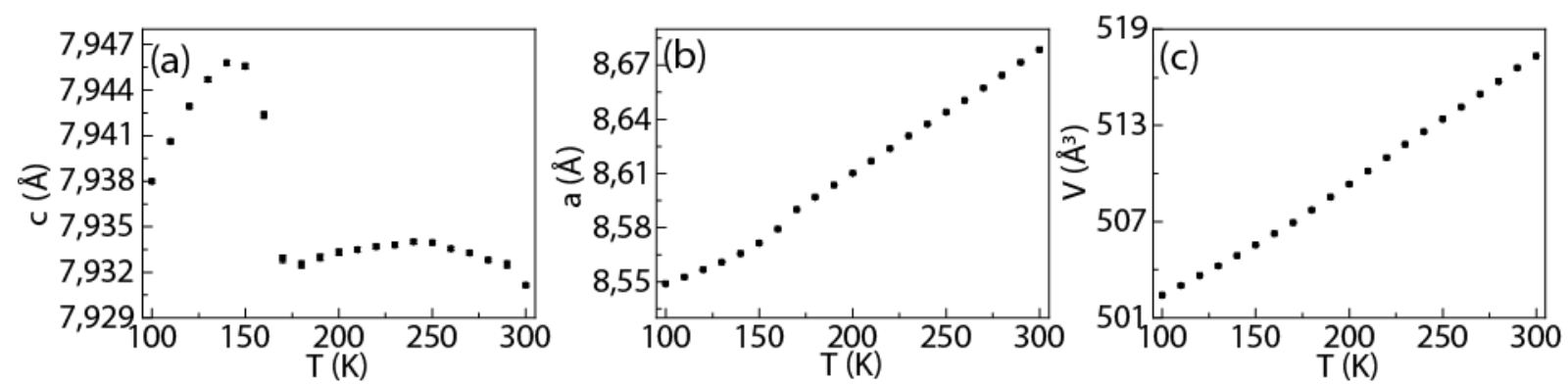

Figure S7: Lattice evolution from PXRD of (a) the $c$-axis, (b) the $a$-axis, and (c) the unit cell volume.

\section{FAPbBr 3 - Supporting information}

\section{Temperature-dependent PXRD}

Structural changes in $\mathrm{FAPbBr}_{3}$ were investigated by following the appearance of new reflections in both the PXRD data, as seen in Figure S8, and the single crystal precession maps in Figure S9. From the powder patterns, new reflections were found at 260, 250, 160, 150, and $140 \mathrm{~K}$. A full list of the new peaks and their position can be seen in Table SII. This is in very good agreement with the phase transitions observed in the dilatometry measurements in Figure 3a. Due to the very low intensity of these reflections, it is difficult to determine at what temperature when they appear. While dilatometry indicates a phase transition at $152 \mathrm{~K}$, most of the new reflections from PXRD appear at $140 \mathrm{~K}$. This can be explained by a small error in the sample temperature when measuring in combination with the transition being very close to $150 \mathrm{~K}$. This results in a shift of $10 \mathrm{~K}$ and the PXRD is therefore consistent with the phase transitions in Table II. No new reflections were observed at $120 \mathrm{~K}$ and below, which could be due to overlap with the peaks stemming from $\mathrm{PbBr}_{2}$ impurities. 


\section{WILEY-VCH}

Table SII: List of the new reflections observed at specific temperatures based on the PXRD patterns in Figure S8.

\begin{tabular}{llllll}
\hline$T(\mathrm{~K})$ & 260 & 250 & 160 & 150 & 140 \\
\hline $2 \theta$ (degrees) & 9.40 & 8.30 & 8.76 & 6.99 & 9.42 \\
& 8.76 & 6.73 & 7.86 & 6.39 & 7.61 \\
& 8.53 & & 3.60 & 3.37 & 6.20 \\
& 7.28 & & & 3.78 \\
& 7.01 & & & 3.48 \\
& 6.11 & & & \\
5.78 & & & \\
& 3.70 & & & \\
\hline
\end{tabular}

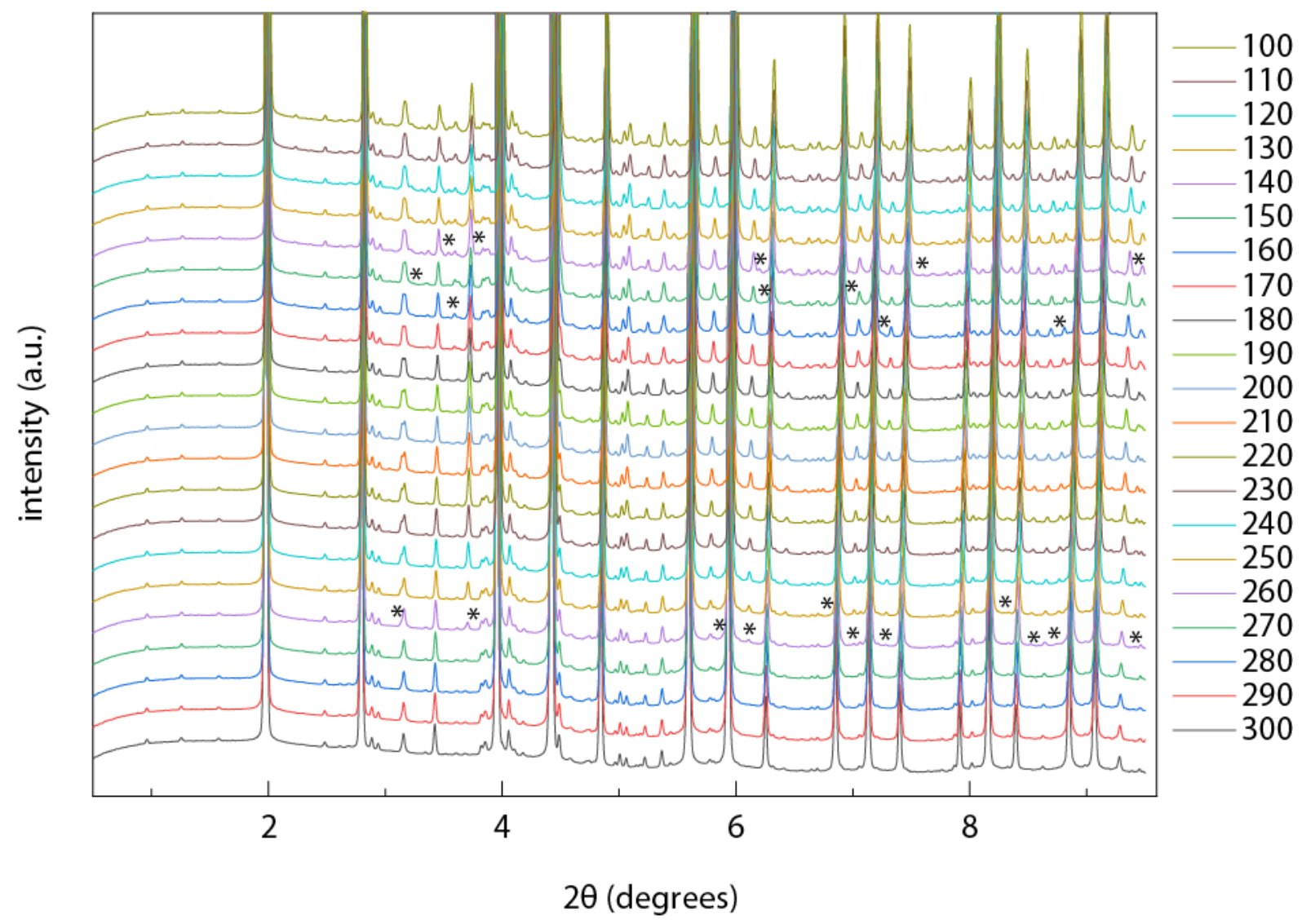

Figure S8. Full temperature range waterfall X-Ray diffraction pattern extracted from PXRD measurements for $\mathrm{FAPbBr} 3$.

\section{Precession maps}

Figure S9 displays precession maps calculated from the small cubic cell with $a \approx 6$ $\AA$ through the first integer- lattice planes of $\mathrm{FAPbBr}_{3}$ at $280 \mathrm{~K}$. The diffraction pattern at $280 \mathrm{~K}$ corresponds to the cubic $\operatorname{Pm} \overline{3} m$ phase. At $250 \mathrm{~K}$ weak reflections start to 


\section{WILEY-VCH}

appear at half-integer values of $h, k$, and $l$ indicating a phase transition. These weak reflections become more numerous and increase in intensity with decreasing temperature. This makes it appear as if the crystal adopts a $2 \times 2 \times 2$ supercell as reflections are found in half-integer lattice planes with respect to the smaller cubic cell, and because all the planes look identical. This inconsistency with the PXRD was explained by the presence of twinning in the crystal after the phase transition. In the Bruker Apex3 software suite two twins are identified rotated $90^{\circ}$ with respect to the first domain, aligning the tetragonal $c$-axes of the two twin domains along the diagonals [110] and [1-10]. In Figure S.10 the $h 1 l$ and $h k 1$ plane at $250 \mathrm{~K}$ calculated from the first domain is illustrated. It can be seen that the general conditions $0 k l: k=2 n$ and $h 00: h=2 n$ from the space group $P 4 / \mathrm{mbm}$ are fulfilled by the absence of reflections in the [01l] direction and at (010). For the $h k l$ lattice plane, moving along the [0k1] direction, it can be seen that every second reflection is absent, verifying the $0 k l: k=2 n$ condition by the presence of the $b$-glide. Additionally, the weak reflections are successfully indexed. Comparing the $d$-spacings in PXRD and SCXRD in Table SIII reveal a perfect match.

Similar analysis was performed for both PXRD and SCXRD at $100 \mathrm{~K}$. The diffraction pattern seemed to adopt a $2 \times 2 \times 2$ cubic cell, whereas an orthorhombic $\sqrt{2} \times$ $2 \times \sqrt{2}$ cell was expected based on the work by Howard and Stokes. ${ }^{[5]}$ Twin analysis revealed a $\sqrt{2} \times 2 \times \sqrt{2}$ pseudocubic cell with two twins rotated $120^{\circ}$ about the [0-11] and [110] axes. This matches with the unit cell configuration for the space group Pnma. A cubic reflection, $h k l$, can be transformed to the orthorhombic axes by $h^{\prime} k^{\prime} l^{\prime}=h+l, 2 k, h-l$. Orthorhombic reflections with $k^{\prime}$ even and $h^{\prime}$ and $l$ ' both even or odd stem from the original small cubic cell. Superlattice reflections should occur for when $k^{\prime}$ is odd and $k^{\prime}$ and $l$ ' not both even or odd. In Figure S11 precession maps through the planes $(1 \mathrm{kl}$ and $h 1 l)$ have been calculated with the orthorhombic cell. In 


\section{WILEY-VCH}

the $1 \mathrm{kl}$ it can be seen, that both the (131) and (155) reflections appear and the systematic absences are compatible with the space group Pnma. Interestingly, when looking through the $h 1 l$ plane it can be seen that almost none of the predicted reflections has intensity. This could be due to the orthorhombic cell pertaining an almost perfect cubic metric which indicate a very small tilt angle of the octahedra. As seen in the PXRD in Figure 3(e), the predicted reflections for $h 1 l$ all show no intensity. The brighter spots which can be seen are described by the twinned domains. The phase transition is far away from the tetragonal symmetry thus appears to be of an extremely subtle nature. All the indexed reflections appeared in the SCXRD are presented in Table SIV. Those indicated by hyphens could not be identified in SCXRD.
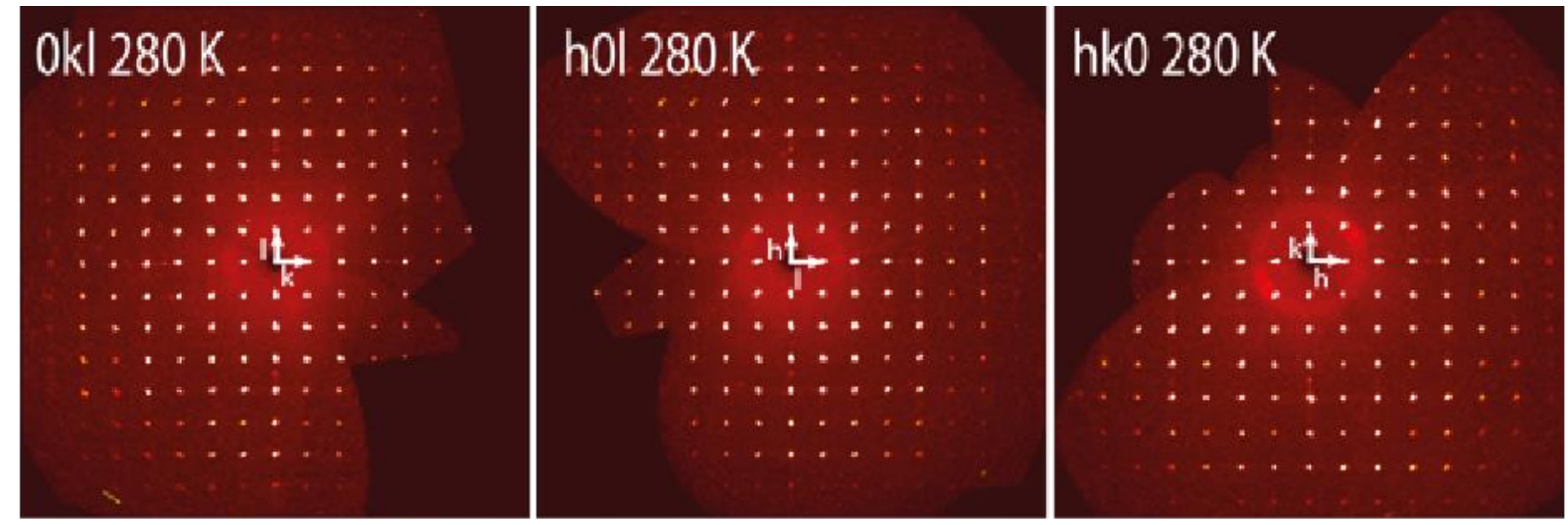

Figure S9. Precession maps calculated from the small cubic cell with $a \approx 6 \AA$ through the first integer lattice planes of $\mathrm{FAPbBr}_{3}$ at $280 \mathrm{~K}$. 


\section{WILEY-VCH}
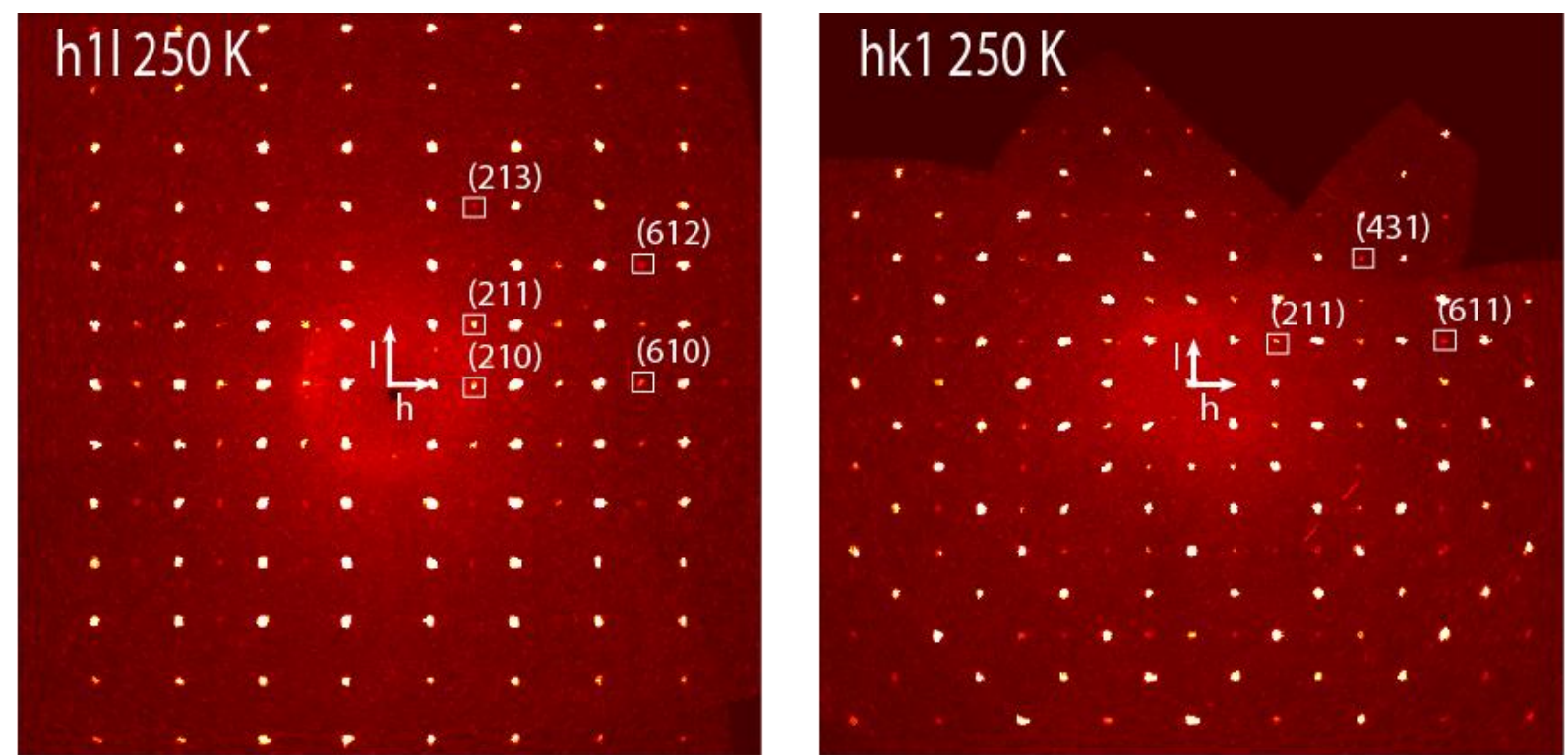

Figure S10. Precession maps calculated from the tetragonal pseudocubic cell through the h11 and hk1 lattice planes at $250 \mathrm{~K}$. Examples of indices matching with the PXRD has been highlighted.

Table SIII. Comparison of the $d$-spacings of the indexed Bragg planes in both PXRD and SCXRD at $250 \mathrm{~K}$.

\begin{tabular}{|c|c|c|c|c|c|c|c|c|c|c|c|c|c|}
\hline$h k l$ & $(210)$ & (211) & $(410)$ & (411) & $(430)$ & (412) & (431) & (213) & (413) & $(610)$ & (611) & (630) & (612) \\
\hline 2theta SCXRD & 8,52 & 10,07 & 15,72 & 16,62 & 19,06 & 19,09 & 19,82 & 18,25 & 22,73 & 23,21 & 23,86 & 25,62 & 25,66 \\
\hline 2theta PXRD & 3,13 & 3,7 & 5,78 & 6,11 & 7,01 & 7,01 & 7,28 & 6,73 & 8,3 & 8,53 & 8,76 & 9,4 & 9,4 \\
\hline d SCXRD $(\AA ⿻)$ & 3,78 & 3,20 & 2,05 & 1,94 & 1,69 & 1,69 & 1,63 & 1,77 & 1,42 & 1,39 & 1,36 & 1,26 & 1,26 \\
\hline d PXRD $(\AA)$ & 3,79 & 3,20 & 2,05 & 1,94 & 1,69 & 1,69 & 1,63 & 1,76 & 1,43 & 1,39 & 1,35 & 1,26 & 1,26 \\
\hline
\end{tabular}
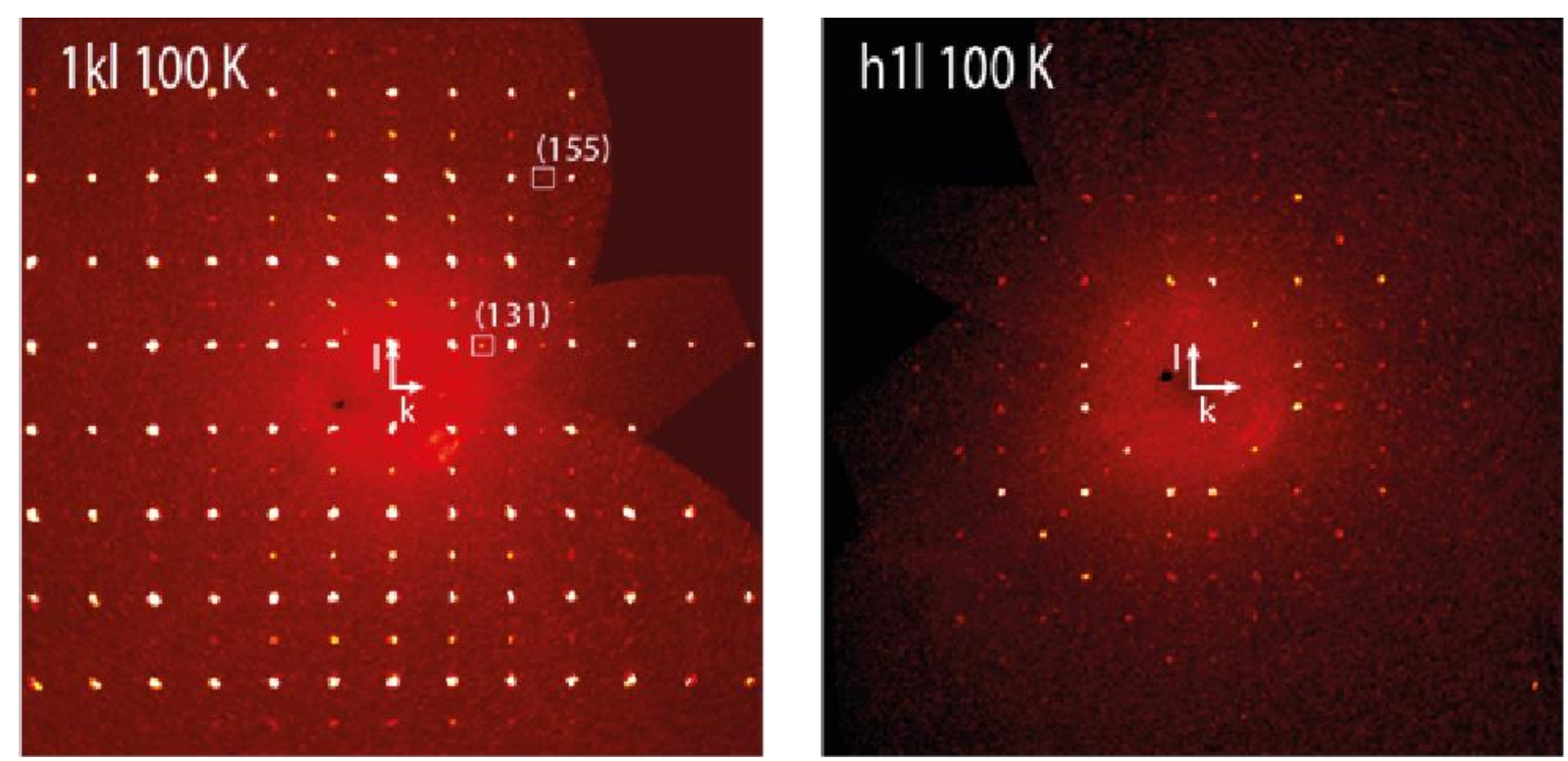

Figure S11. Precession maps calculated from the orthorhombic pseudocubic cell through the $h 1 l$ and $h k 1$ lattice planes at $100 \mathrm{~K}$. Examples of indices matching with the PXRD has been highlighted. 


\section{WILEY-VCH}

Table SIV. Comparison of the $d$-spacings of the indexed Bragg planes in both PXRD and SCXRD at $100 \mathrm{~K}$.

\begin{tabular}{cccccccc}
\hline$h k l$ & $(131)$ & $(214 / 252 / 430)$ & $(234)$ & $(205 / 502)$ & $(522 / 362 / 263 / 225)$ & $(533 / 155 / 335)$ & $(236 / 274 / 454 / 290)$ \\
\hline 2theta SCXRD & 9,79 & Not observed & 19,06 & Not observed & Not observed & 24,02 & Not observed \\
\hline 2theta PXRD & 3,59 & 6,39 & 6,99 & 7,61 & 7,86 & 8,76 & 9,42 \\
\hline d scxrd $(\AA)$ & 3,29 & - & 1,69 & - & - & 1,35 & - \\
\hline d pxrd $(\AA)$ & 3,30 & 1,86 & 1,70 & 1,56 & 1,51 & 1,35 & 1,26 \\
\hline
\end{tabular}

\section{References}

[S1] P. Fassl, V. Lami, A. Bausch, Z. Wang, M. T. Klug, H. J. Snaith and Y. Vaynzof, Energy Environ. Sci., 2018, 11, 3380

[S2] A. Dobrovolsky, A. Merdasa, E. L. Unger, A. Yartsev \& I. G. Scheblykin, Nature Communications, 2017, 8, Article number: 34.

[S3] N. Boens and M. Van der Auweraer, Photochem. Photobiol. Sci., 2014, 13, 422.

[S4] T. Chen, B. J. Foley, C. Park, C. M. Brown, L. W. Harriger, J. Lee, J. Ruff, M. Yoon, J.J. Choi, Seung-Hun Lee, Science Advances, 2016, 160, 1650.

[S5] C. J. Howarda and H. T. Stokes, Acta Cryst. 1998, B54, 782-789. 